\title{
Finding 2-Edge and 2-Vertex Strongly Connected Components in Quadratic Time*
}

\author{
Monika Henzinger $^{\dagger} \quad$ Sebastian Krinninger ${ }^{\dagger} \quad$ Veronika Loitzenbauer $^{\dagger}$
}

\begin{abstract}
We present faster algorithms for computing the 2-edge and 2-vertex strongly connected components of a directed graph. While in undirected graphs the 2-edge and 2-vertex connected components can be found in linear time, in directed graphs with $m$ edges and $n$ vertices only rather simple $O(m n)$-time algorithms were known. We use a hierarchical sparsification technique to obtain algorithms that run in time $O\left(n^{2}\right)$. For 2-edge strongly connected components our algorithm gives the first running time improvement in 20 years. Additionally we present an $O\left(\mathrm{~m}^{2} / \log n\right)$-time algorithm for 2-edge strongly connected components, and thus improve over the $O(m n)$ running time also when $m=O(n)$. Our approach extends to $k$-edge and $k$-vertex strongly connected components for any constant $k$ with a running time of $O\left(n^{2} \log n\right)$ for $k$-edge-connectivity and $O\left(n^{3}\right)$ for $k$-vertex-connectivity.
\end{abstract}

\section{Introduction}

Problem Description. In a directed graph $G$ two vertices $u$ and $v$ are 2-edge strongly connected if from $u$ to $v$ and from $v$ to $u$, respectively, there are two paths that have no common edge. A 2-edge strongly connected component (2eSCC) of $G$ is a maximal subgraph of $G$ such that in the subgraph every pair of distinct vertices is 2-edge strongly connected. Two vertices $u$ and $v$ are 2-vertex strongly connected in $G$ if they remain strongly connected after the removal of any single vertex except $u$ and $v$ from $G$. A 2-vertex strongly connected component (2vSCC) of $G$ is a maximal subgraph of $G$ such that in the subgraph every pair of distinct vertices is 2-vertex strongly connected. Edge and vertex connectivity are central properties of graphs and have many applications [BJG09, NI08], for example in the construction of reliable communication networks [BM76] and in the analysis of the structure of networks [New10].

Our Results. In this work we present algorithms that compute the 2eSCCs and the 2vSCCs of a directed graph in $O\left(n^{2}\right)$ time. For 2eSCCs we additionally provide an algorithm that runs in $O\left(m^{2} / \log n\right)$ time, which is faster than $O\left(n^{2}\right)$ if $m=O(n)$. Thus we significantly

\footnotetext{
${ }^{*}$ A preliminary version of this paper is presented at the 42 2nd International Colloquium on Automata, Languages, and Programming (ICALP 2015).

${ }^{\dagger}$ University of Vienna, Faculty of Computer Science, Austria. This work was supported by the Austrian Science Fund (FWF): P23499-N23. Additionally, the research leading to these results has received funding from the European Research Council under the European Union's Seventh Framework Programme (FP/2007-2013) / ERC Grant Agreement no. 340506.
} 
improve upon the previous $O(m n)$-time algorithms for both 2eSCCs [NW93, $\mathrm{GIL}^{+} 15 \mathrm{a}$ ] and 2vSCCs [Jab14]. For 2eSCCs the previous upper bound stood for 20 years. Our approach immediately generalizes to computing the $k$-edge strongly connected components (keSCCs) and the $k$-vertex strongly connected components (kvSCCs). We give algorithms that, for any integral constant $k>2$, compute (1) the keSCCs in time $O\left(n^{2} \log n\right)$ (improving upon the previous upper bound of $O(m n)$ [NW93]) and (2) the kvSCCs in time $O\left(n^{3}\right)$ (improving upon the previous upper bound of $O\left(m n^{2}\right)$ [Mak88]).

Related Work. The 2-edge and 2-vertex connected components of an undirected graph can be determined in linear time [Tar72, HT73]. In directed graphs several related problems can be solved in linear time: Testing whether a graph is 2-edge or 2-vertex strongly connected [Tar76, GT85, Geo10], finding all strong bridges and strong articulation points [ILS12], and determining the 2-edge and 2-vertex strongly connected blocks [GIL $\left.{ }^{+} 15 \mathrm{a}, \mathrm{GIL}^{+} 15 \mathrm{~b}\right]$. An edge is a strong bridge and a vertex is a strong articulation point, respectively, if its removal from the graph increases the number of strongly connected components (SCCs) of the graph. Note the difference between 'blocks' and 'components' in directed graphs: In a 2-edge strongly connected block every pair of distinct vertices is 2-edge strongly connected; however, as opposed to a $2 \mathrm{eSCC}$, the paths to connect the vertices in a block might use vertices that are not in the same block. Each $2 \mathrm{eSCC}$ is completely contained in one 2-edge strongly connected block, i.e., the 2eSCCs refine the 2-edge strongly connected blocks. In Appendix $\mathrm{C}$ we provide a construction that shows that knowing the 2-edge strongly connected blocks of a graph does not help in finding its 2eSCCs. The relation between blocks and components for vertex connectivity is analogous.

Georgiadis et al. [GIL ${ }^{+} 15 \mathrm{a}$ ] and Jaberi [Jab14] described simple algorithms to compute the 2eSCCs and 2vSCCs in $O(m n)$-time, respectively, and posed as an open problem whether this can be improved to linear time as well. An $O(m n)$ running time for computing the 2eSCCs was already achieved by Nagamochi and Watanabe in 1993 [NW93], which in fact solved the more general problem of computing the keSCCs. To the best of our knowledge, the fastest known algorithm for computing the kvSCCs is by Makino [Mak88] and has a running time of $O\left(m n^{2}\right.$ ) (when combined with an $O(m n)$-time algorithm for finding minimum vertex-separators [Eve75, Gal80, HRG00, Gab06]; combined with [Geo10] and [BGK ${ }^{+} 08$ ] it also gives an $O(m n)$-time algorithm for $2 \mathrm{vSCCs})$. In undirected graphs there are lineartime algorithms for computing both the 3-edge [GI91] and the 3-vertex [HT73] connected components. The $k$-edge connected components of an undirected graph can be computed in time $O\left(n^{2}\right)$ [NW93]. The runtime of Makino's algorithm can for $k$-vertex connected components in undirected graphs be reduced to $O\left(n^{3}\right)$ by a preprocessing step [NI92]. Thus, to the best of our knowledge, our algorithms for keSCCs and kvSCCs match the runtimes for undirected graphs for $k>3$ (up to a logarithmic factor).

Techniques. We use a hierarchical graph sparsification that was introduced by Henzinger et al. [HKW99] for undirected graphs and extended to directed graphs and game graphs in [CH14, CHL15]. Roughly speaking, this sparsification technique allows us to replace the ' $m$ ' in the $O(m n)$ running time by an ' $n$ ', yielding $O\left(n^{2}\right)$. Our main technical contribution is to find structural properties of connectivity in directed graphs that allow us to apply this technique. Note that while various ways of sparsification are used in algorithms 
for undirected graphs, such approaches are rarely found for directed graphs. For example, Georgiadis et al. [GIL ${ }^{+} 15 \mathrm{a}$ ] showed that a sparse certificate for 2-edge strong connectivity can be constructed in $O(m)$ time; the sparsification framework by Eppstein et al. [EGI ${ }^{+} 97$ ] would yield a dynamic algorithm with $O(n)$ update time if a sparse strong certificate could be constructed in $O(m)$ time; however, Khanna et al. [KMW98] showed that even a strong certificate for strong connectivity has size $\Omega(m)$, i.e., is not sparse. We therefore strongly believe that understanding how to apply sparsification for directed graphs is interesting in its own right.

We briefly present the main ideas behind our algorithm for 2-vertex connectivity. The approach for edge connectivity is similar. The fastest known asymptotic running time of $O(m n)$ for computing $2 \mathrm{vSCCs}$ can be achieved with the following approach: Assume that the graph is strongly connected. First find a strong articulation point of the graph, i.e., a vertex whose removal increases the number of SCCs. Then remove the strong articulation point and compute the SCCs. For each SCC, recurse on the subgraph it induces together with the strong articulation point. The recursion stops when no strong articulation point is found anymore. The SCCs remaining in the end are the 2vSCCs. We now explain in which way our algorithm deviates from this scheme.

Let for 2-vertex connectivity a 2-isolated set $S$ be a set of vertices that (a) cannot be reached by the vertices of $V \backslash S$ or (b) that can be reached from $V \backslash S$ only through one vertex $v$. We show that every 2 vSCC of $G$ contains either only vertices of $S \cup\{v\}$ or only vertices of $V \backslash S$. Thus the algorithm can recurse on the subgraphs induced by $S \cup\{v\}$ and $V \backslash S$, respectively. The difference to the straightforward approach is thus the following: Instead of repeatedly identifying strong articulation points, we focus on separating 2-isolated sets of vertices. To see why this is useful, note that the incoming edges of the vertices of a 2 -isolated set $S$ consist of the incoming vertices from other vertices of $S$ and edges from at most one vertex of $V \backslash S$ to $S$. Thus the number of incoming edges of each vertex in $S$ is bounded by the number of vertices in $S$. We use this insight as follows: When searching for a 2-isolated set, we start the search in a subgraph of $G$ that includes all vertices but only the first incoming edge of each vertex. If no 2 -isolated set is found, we repeatedly double the number of incoming edges per vertex in the subgraph until the search is successful. In this way the search will take time $O(n)$ per vertex in the 2-isolated set. This will allow us to bound the total runtime by $O\left(n^{2}\right)$. Note that to achieve this running time we cannot afford to compute all SCCs in each recursive call because the recursion depth might be $\Theta(n)$; we therefore do not assume that the input graph is strongly connected.

To correctly identify 2 -isolated sets by a search in a proper subgraph of $G$, the algorithm finds vertex-dominators in slightly modified flow graphs. A flow graph is a directed graph with a designated root where all vertices are reachable from the root. A vertex is a vertexdominator in a flow graph if some other vertex can be reached from the root only through this vertex. Our algorithms use the linear-time algorithms for finding dominators [Tar76, GT85, $\left.\mathrm{BGK}^{+} 08\right]$ and SCCs [Tar72] as subroutines.

In the $O\left(m^{2} / \log n\right)$-algorithm for 2eSCCs we search for 2-(edge-)isolated sets in subgraphs that are obtained by local breadth-first searches from vertices that lost edges in the previous iteration of the algorithm. Such local breadth-first searches were first used for Büchi games by Chatterjee et al. [CJH03]. 
Outline. In the main part of the paper we describe the result for 2 vSCCs in more detail. In Section 2 the main definitions and the notation are introduced. In Section 3 we show when and how we can identify a 2 -isolated set in a proper subgraph of $G$. In Section 4 we present the $O\left(n^{2}\right)$-algorithm for 2vSCCs. In Section 5 we outline how the results from Sections 3 and 4 extend to keSCCs and kvSCCs. In Appendix A we provide a full version with all proofs for keSCCs and kvSCCs. In Appendix B we present the $O\left(m^{2} / \log n\right)$-algorithm for 2eSCCs.

\section{Preliminaries}

Let $G=(V, E)$ be a directed graph with $m=|E|$ edges and $n=|V|$ vertices. Except when mentioned explicitly, we only consider simple graphs, i.e., graphs without parallel edges. The reverse graph $\operatorname{Rev}(G)$ of $G$ is equal to $\left(V, E^{R}\right)$ where $E^{R}$ is the set containing for each edge $(u, v) \in E$ its reverse $(v, u)$. We use $S \subseteq V$ to denote a subset $S$ of $V$ and $S \subsetneq V$ to denote a proper subset $S$ of $V$. For any set $S \subseteq V$ we denote by $G[S]$ the subgraph of $G$ induced by the vertices in $S$, i.e., the graph $(S, E \cap(S \times S))$. We call edges from some $u \in V \backslash S$ to some $v \in S$ the incoming edges of $S$. The incoming edges of a vertex $v$ in $G$ are denoted by $\operatorname{In}_{G}(v)$, the number of incoming edges by $\operatorname{Indeg}_{G}(v)$; analogously we use $\operatorname{Out}_{G}(v)$ and $\operatorname{Outdeg}_{G}(v)$ for outgoing edges. We denote by $G \backslash V^{\prime}$ the graph $G\left[V \backslash V^{\prime}\right]$ and by $G \backslash E^{\prime}$ the graph $\left(V, E \backslash E^{\prime}\right)$ for an arbitrary set of vertices $V^{\prime} \subseteq V$ and an arbitrary set of edges $E^{\prime} \subseteq E$.

Strong Connectivity. A subgraph $G[S]$ induced by some set of vertices $S$ is strongly connected if for every pair of distinct vertices $u$ and $v$ in $S$ there exists a path from $u$ to $v$ and a path from $v$ to $u$ in $G[S]$. A single vertex is considered strongly connected. The strongly connected components (SCCs) of $G$ are its maximal strongly connected subgraphs and form a partition of $V$. A strongly connected subgraph with no outgoing edges is a bottom $S C C$ (bSCC), a strongly connected subgraph with no incoming edges is a top $S C C$ (tSCC). By definition, bSCCs and tSCCs are maximal. Every graph $G$ contains at least one bSCC and at least one tSCC. If $G$ is not strongly connected, then there exist both a bSCC and a tSCC that are disjoint and thus one of them contains at most half of the vertices of $G$. Note that a bSCC in $G$ is a tSCC in $\operatorname{Rev}(G)$ and vice versa. We further use that when a set of vertices $S$ cannot be reached by any vertex of $V \backslash S$ in $G$, then $G[S]$ contains a tSCC of $G$. The SCCs of a graph can be computed in $O(m)$ time [Tar72].

Strong 2-Vertex Connectivity. A vertex $v \in V$ is a strong articulation point if the removal of $v$ from $G$ increases the number of SCCs in $G$. All strong articulation points of a graph can be found in time $O(m)$ [ILS12]. Two (simple) paths are internally vertex-disjoint if they do not share a vertex except possibly their endpoints. Two distinct vertices $u$ and $v$ are 2-vertex strongly connected in $G$ if they are strongly connected and remain strongly connected after the removal of any vertex except $u$ and $v$ from $G$. If there is no edge between $u$ and $v$, then it holds that $u$ and $v$ are 2-vertex strongly connected if and only if there exists two internally vertex-disjoint paths from $u$ to $v$ and two internally vertex-disjoint paths from $v$ to $u$ [GIL ${ }^{+} 15 \mathrm{a}$ ]. A subgraph $G[S]$ induced by some set of vertices $S$ is 2-vertex strongly connected if every pair of distinct vertices $u$ and $v$ in $S$ is 2-vertex strongly connected 


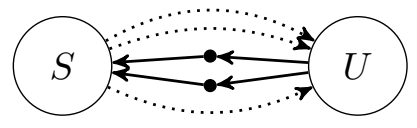

in $G[S]$. The 2-vertex strongly connected components ${ }^{1}$ (2vSCCs) of a graph are its maximal 2 -vertex strongly connected subgraphs. Equivalently, the 2vSCCs are the maximal strongly connected subgraphs such that none of the subgraphs contains a strong articulation point. This definition of 2vSCCs allows for degenerate 2vSCCs with less than three vertices. While the 2eSCCs form a partition of the vertices of the graph, the 2vSCCs form a partition of a subset of the edges. In the remainder of the paper we omit "strong(ly)" from the above definitions whenever it is clear from the context.

Flow Graphs. We define the flow graph $G(r)$ to be the graph $G$ with a vertex $r \in V$ designated as the root and with all vertices not reachable from $r$ removed. A vertex-dominator in $G(r)$ is a vertex $v \in V \backslash\{r\}$ for which there exists a vertex $u \in V \backslash\{r, v\}$ such that $u$ is reachable from $r$ and every path from $r$ to $u$ contains $v$. We say that $v$ dominates $u$ in $G(r)$. Note that in contrast to articulation points the removal of a vertex-dominator from $G$ might not increase the number of SCCs but instead might remove edges between SCCs. The vertex-dominators of a flow graph can be computed in linear time [BGK $\left.{ }^{+} 08\right]$.

\section{New top SCCs and dominators in subgraphs}

Let an isolated set $S$ w.r.t. 2-vertex-connectivity (2-isolated set) be a set of vertices with (1) incoming edges from at most one vertex and for which (2) there exist vertices without edges to $S$ in $G$. 2-isolated sets can be used to design a divide-and-conquer based algorithm for the following reason: Let $T$ be the vertex set of a $2 \mathrm{vSCC}$. The $2 \mathrm{vSCC} G[T]$ is (1) strongly connected and (2) for any proper subset $S$ of $T$ such that there exists a set of vertices $U$ in $T$ that has no edge to any vertex of $S$, there are at least two vertices in $T \backslash(S \cup U)$ that connect $U$ with the vertices in $S$. Thus if we detect a set of vertices $S$ that (a) cannot be reached by the vertices of $V \backslash S$ or (b) that can be reached from $V \backslash S$ only through one vertex $v$, then we know that each $2 \mathrm{vSCC}$ of $G$ contains either only vertices of $S \cup\{v\}$ or only vertices of $V \backslash S$. A 2-isolated set satisfies (a) or (b). Our algorithm repeatedly identifies specific 2-isolated sets $S$ and recurses on the subgraphs induced by $S \cup\{v\}$ and $V \backslash S$, respectively. As the recursion depth can be $\Theta(n)$, to achieve an $o(m n)$ running time, we cannot afford to look at all edges in each level of recursion. Thus our algorithms are based on the following question: Can we identify 2-isolated sets by searching in a proper subgraph of $G$ ? Note that whenever an articulation point $v$ is removed from a strongly connected graph $G$, then there exist both a tSCC and a bSCC in $G \backslash\{v\}$ that were adjacent to $v$ in $G$ and are disjoint. Let $T$ be the vertices in the tSCC in $G \backslash\{v\}$. Observe that $T$ is a 2 -isolated set in $G$. Further, if $T$ contains only a few vertices, then each vertex in $T$ has a low in-degree in $G$ because all incoming edges to vertices in $T$ in $G$ come from $v$ or other vertices of $T$. In our algorithm we search for such "almost tSCCs" $G[T]$ in the subgraph of $G$ induced by vertices with low

\footnotetext{
${ }^{1}$ Our definitions follow [GIL $\left.{ }^{+} 15 \mathrm{a}\right]$, while $\left[\mathrm{Jab} 14, \mathrm{GIL}^{+} 15 \mathrm{~b}\right]$ use slightly different definitions. The 2vSCCs of $\left[\mathrm{Jab} 14, \mathrm{GIL}^{+} 15 \mathrm{~b}\right]$ can be determined in $O(n)$ time from the 2vSCCs defined here.
} 
in-degree, which only takes time linear in the number of edges in this subgraph. We do the same on $\operatorname{Rev}(G)$ to detect small almost bSCCs.

Definition 3.1. A set of vertices $T$ induces an almost tSCC in $G$ with respect to a vertex $v$ if $G[T]$ is a $t S C C$ in $G \backslash\{v\}$ but has incoming edges from $v$ in $G$.

Given a vertex $v$ such that an almost tSCC induced by $T$ w.r.t. $v$ exists, the top SCC $G[T]$ can be identified in a subgraph of $G \backslash\{v\}$ in time linear in the number of edges in the subgraph as long as it is contained in the subgraph. But how can we identify the vertex $v$ without looking at the whole graph? Assume there exists a vertex $r \neq v$ that is not in $T$ but can reach $v$. Since $G[T]$ is a tSCC in $G \backslash\{v\}$, it follows that $v$ dominates every vertex of $T$ in the flow graph $G(r)$. This still holds in any subgraph of $G$ as long as $r$ can reach $T$ in the subgraph. If additionally all incoming edges of the vertices in $T$ are present in the subgraph, we can identify $v$ and $T$ in time linear in the number of edges in the subgraph by finding the vertex-dominator $v$ in the flow graph with root $r$ and the tSCC $G[T]$ in the subgraph with $v$ removed. Thus, instead of finding the right $v$, we only have to find the right $r$. As edges are missing in the subgraph, it is not a-priori clear how to choose $r$, but, as shown below, we can use an artificial vertex as root $r$. Hence our approach is to first search for vertex-dominators $v$ in a subgraph with an additional artificial root and then for a tSCC in the subgraph with $v$ removed. When the search is successful, we recurse separately on the almost tSCC and the remaining graph.

In our algorithm we cannot afford to identify all SCCs in the current graph $G$ as we only want to spend time proportional to the edges in a proper subgraph of $G$; thus we cannot assume that the graph we are considering is strongly connected. This means that, in contrast to strongly connected graphs [ILS12], when we identify a vertex-dominator $v$ in $G(r)$, the vertex $v$ might not necessarily be an articulation point in $G$. However, for an almost tSCC w.r.t. $v$ we still know that the set of vertices $T$ in the almost tSCC is a 2-isolated set, i.e., all vertices of $V \backslash(T \cup\{v\})$ that can reach $T$ in $G$ can reach $T$ only through $v$. Thus there cannot be two internally vertex-disjoint paths from any vertex of $G \backslash(T \cup\{v\})$ to any vertex of $T$. This intuition about almost tSCCs is summarized in the following lemma, which we use to show the correctness of our approach.

Lemma 3.2. Let $v$ be a vertex such that some set of vertices $T$ induces an almost $t S C C$ with respect to $v$ in $G$. Let $W=V \backslash(T \cup\{v\})$. If $W \neq \emptyset$, then there do not exist two internally vertex-disjoint paths from any vertex of $W$ to any vertex of $T$ in $G$, i.e., no vertex of $W$ is 2-vertex-connected to any vertex of $T$. Additionally, the vertex $v$ is a vertex-dominator in $G(r)$ for every $r \in W$ that can reach $v$ in $G$.

Let $G_{h}=\left(V_{h}, E_{h}\right)$ be a subgraph of a directed graph $G=(V, E)$, i.e., $V_{h} \subseteq V$ and $E_{h} \subseteq G\left[V_{h}\right]$. We use the index $h$ to identify specific subgraphs. In the remainder of this section we want to characterize which almost tSCCs in $G$ we can identify in $G_{h}$. Let $v$ be a vertex such that an almost tSCC w.r.t. $v$ exists in $G$. To identify $v$ as a vertex-dominator in a flow graph, we define below a graph created from $G_{h}$ with an auxiliary root. Let the white vertices $A_{G, h} \subseteq V_{G}$ be the set of vertices for which we have the guarantee that for each vertex in $A_{G, h}$ its incoming edges in $G_{h}$ are the same as in $G$. Let $B_{G, h}=V_{h} \backslash A_{G, h}$ be the blue vertices, which might miss incoming edges in $G_{h}$ compared to $G$. We show that as long as the vertices in the almost tSCC are white, i.e., are not missing incoming edges 
in $G_{h}$, an almost tSCC w.r.t. a vertex $v$ in $G_{h}$ is an almost tSCC w.r.t. $v$ in $G$ and vice versa. In contrast, no conclusions can be drawn from an almost tSCC in $G_{h}$ that includes blue vertices.

Definition 3.3. For a given subgraph $G_{h}=\left(V_{h}, E_{h}\right)$ of a directed graph $G=(V, E)$ and a set of blue vertices $B_{G, h}$ that contains all vertices that have fewer incoming edges in $G_{h}$ than in $G$, we define the flow graph $F_{G, h}\left(r_{G, h}\right)$ as follows. If $\left|B_{G, h}\right| \geq 2$, let $F_{G, h}$ be the graph $G_{h}$ with an additional vertex $r_{G, h}$ and an additional edge from $r_{G, h}$ to each vertex in $B_{G, h}$. If $B_{G, h}$ contains a single vertex, we name it $r_{G, h}$ and let $F_{G, h}=G_{h}$.

Note that if we would define the flow graph $F_{G, h}\left(r_{G, h}\right)$ in the case $\left|B_{G, h}\right|=1$ in the same way as for $\left|B_{G, h}\right|>1$, then in $F_{G, h}\left(r_{G, h}\right)$ the root $r_{G, h}$ could reach the vertices in $A_{G, h}$ only through the vertex in $B_{G, h}$, i.e., the vertex in $B_{G, h}$ would be a vertex-dominator in $F_{G, h}\left(r_{G, h}\right)$ independent of the underlying graph $G$. In the following consider a subgraph $G_{h}$ and a set of vertices $V_{h}$ partitioned into $B_{G, h}$ and $A_{G, h}$ as defined above; the statements for $F_{G, h}$ hold whenever $F_{G, h}$ is defined.

Lemma 3.4. A set of white vertices $T \subseteq A_{G, h}$ induces a $t S C C$ in $G_{h}$ and $F_{G, h}$, respectively, if and only if it induces a $t S C C$ in $G$.

If white vertices $T$ induce an almost $\operatorname{tSCC} G[T]$ with respect to $v$, all incoming edges, and thus $v$, are present in $G_{h}$. This implies the following corollary.

Corollary 3.5. A set of white vertices $T \subseteq A_{G, h}$ induces an almost $t S C C$ with respect to a vertex $v \in V$ in $G_{h}$ and $F_{G, h}$, respectively, if and only if it induces an almost $t S C C$ with respect to $v$ in $G$.

The following lemma specifies which almost tSCCs w.r.t. a vertex $v$ in $G$ we can identify by searching for vertex-dominators in $F_{G, h}\left(r_{G, h}\right)$ based on the reachability of $v$ from the vertices in $B_{G, h}$.

Lemma 3.6. Assume $B_{G, h} \neq \emptyset$, let $T \subseteq A_{G, h}$ be a set of white vertices, and let $v \in V$ be such that there exists an almost $t S C C G[T]$ with respect to $v$ in $G$. If $v$ is either not in $B_{G, h}$ and can be reached from a vertex of $B_{G, h}$ or $v$ is in $B_{G, h}$ and $\left|B_{G, h}\right| \geq 2$, then $v$ is a dominator in $F_{G, h}\left(r_{G, h}\right)$.

In the following section we define specific subgraphs $G_{h}$ that allow us to identify an almost tSCC in $G$ that has at most a certain size by searching for vertex-dominators $v$ in $F_{G, h}\left(r_{G, h}\right)$ and tSCCs in $G_{h} \backslash\{v\}$. We additionally have to consider one special case, namely if $v$ is the only vertex in $B_{G, h}$ and an almost tSCC w.r.t. $v$ exists. In this case we have $r_{G, h}=v$. We explicitly identify almost tSCCs with respect to this vertex.

\section{$42 \mathrm{vSCCs}$ in $O\left(n^{2}\right)$ time}

In this section we provide some intuition for the algorithm and outline its analysis. All proofs are given in Appendix A. To find vertex-dominators, articulation points, and SCCs the known linear time algorithms are used (see Section 1).

Let $G=(V, E)$ be a simple directed graph. We consider for $i \in \mathbb{N}$ the subgraphs $G_{i}=\left(V, E_{i}\right)$ of $G$ where $E_{i}$ contains for each vertex of $V$ its first $2^{i}$ incoming edges in $E$ 
(for some arbitrary but fixed ordering of the incoming edges of each vertex). Note that when $i \geq \log \left(\max _{v \in V} \operatorname{Indeg}_{G}(v)\right)$, then $G_{i}=G$. Let $\gamma$ be the minimum of $\max _{v \in V} \operatorname{Indeg}_{G}(v)$ and $\max _{v \in V} \operatorname{Outdeg}_{G}(v)$. Following Definition 3.3, the set $B_{G, i}$ contains all vertices with in-degree more than $2^{i}$ in $G$.

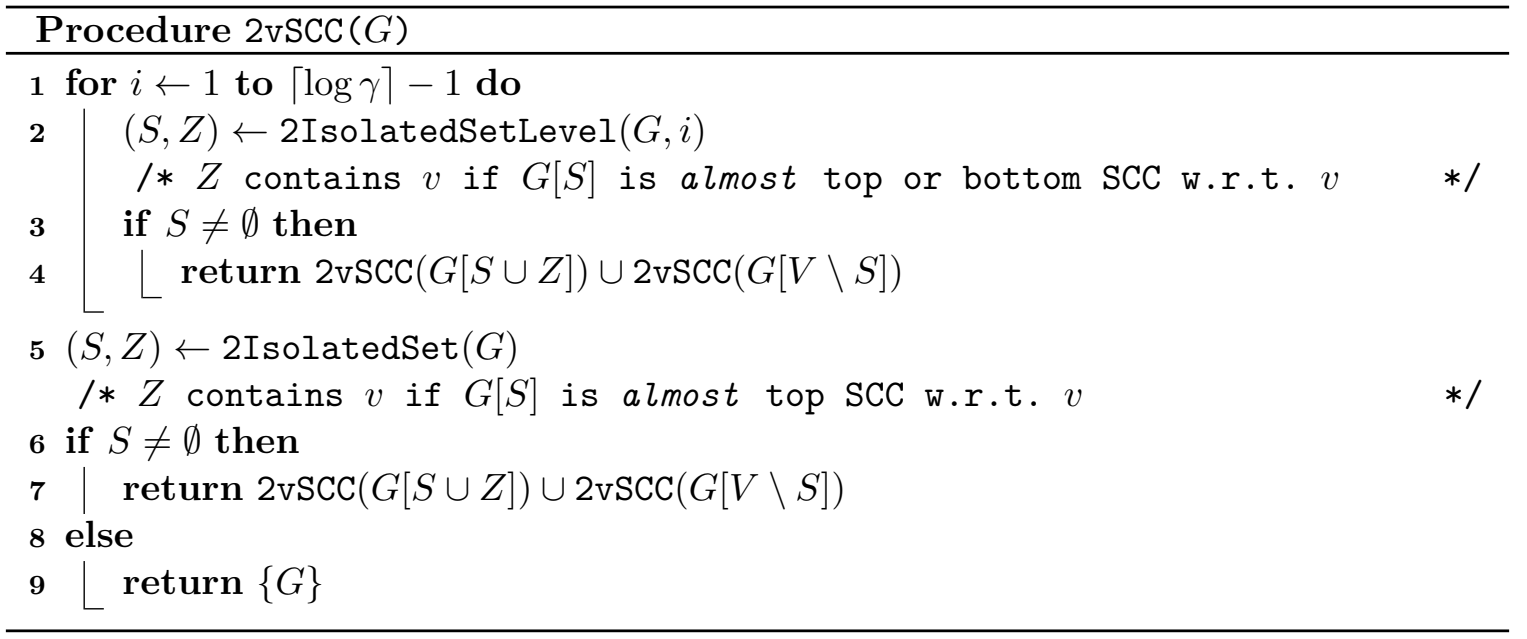

Let $S$ be a set of at most $2^{i}$ vertices that induces a strongly connected subgraph $G[S]$ of $G$ such that $G[S]$ is a top SCC or an almost top SCC with respect to some vertex $v$. Since the only edges from vertices of $V \backslash S$ to $S$ are from $v$, the in-degree of each vertex in $S$ can be at most $2^{i}$. By applying the results from the previous section, we show that we can detect such a set $S$ by searching for SCCs and vertex-dominators in the graphs $F_{G, i}$ constructed from $G_{i}$ with the artificial root $r_{G, i}$ as in Definition 3.3.

Lemma 4.1. If a set of vertices $S$ with $|S| \leq 2^{i}$ induces a tSCC or an almost $t S C C$ in $G$ with respect to some vertex $v$, then $S \subseteq V \backslash B_{G, i}$.

To find bSCCs and almost bSCCs we also search for top SCCs in $\operatorname{Rev}(G)$. The search for both top and bottom SCCs ensures that whenever an (almost) tSCC and a disjoint (almost) bSCC exist in $G$, we only spend time proportional to the smaller one. This search is performed in Procedure 2IsolatedSetLevel, which fulfills the following guarantee.

Lemma 4.2. If for some integer $1 \leq i<\log \gamma$ and $\mathcal{G} \in\{G, \operatorname{Rev}(G)\}$ there exists a set of vertices $T \subseteq V \backslash B_{\mathcal{G}, i}$ that induces in $\mathcal{G}$ a $t S C C$ or an almost $t S C C$ with respect to some vertex $v$ with $T \subsetneq V \backslash\{v\}$, then 2 IsolatedSetLevel $(G, i)$ returns a non-empty set $S$.

In Procedure 2vSCC we start the search for (almost) top SCCs at $i=1$. Whenever the search is not successful, we increase $i$ by one, until we have $G_{i}=G$ or $\operatorname{Rev}(G)_{i}=$ $\operatorname{Rev}(G)$. For the search the Procedure 2IsolatedSetLevel is used as long as $2^{i}<\gamma$, i.e., both $B_{G, i}$ and $B_{\operatorname{Rev}(G), i}$ are non-empty, and the Procedure 2IsolatedSet afterwards. Procedure 2IsolatedSet identifies an (almost) top SCC in $G$ if one exists by using the known procedures for finding SCCs and articulation points. In this way we can show that whenever we had to go up to $i^{*}$ or had to use Procedure 2IsolatedSet to identify an (almost) top or bottom SCC in $G$, the identified subgraph contains $\Omega\left(2^{i^{*}}\right)$ vertices, where $i^{*}=\lceil\log \gamma\rceil$ for Procedure 2IsolatedSet. This will imply that the search in $G_{i}$ and $\operatorname{Rev}(G)_{i}$ for $i$ up to 
$i^{*}$ takes time $O\left(n \cdot 2^{i^{*}}\right)$ which is $O(n \cdot \min \{|S|,|V \backslash S|\})$. This will allow us to bound the total running time by $O\left(n^{2}\right)$.

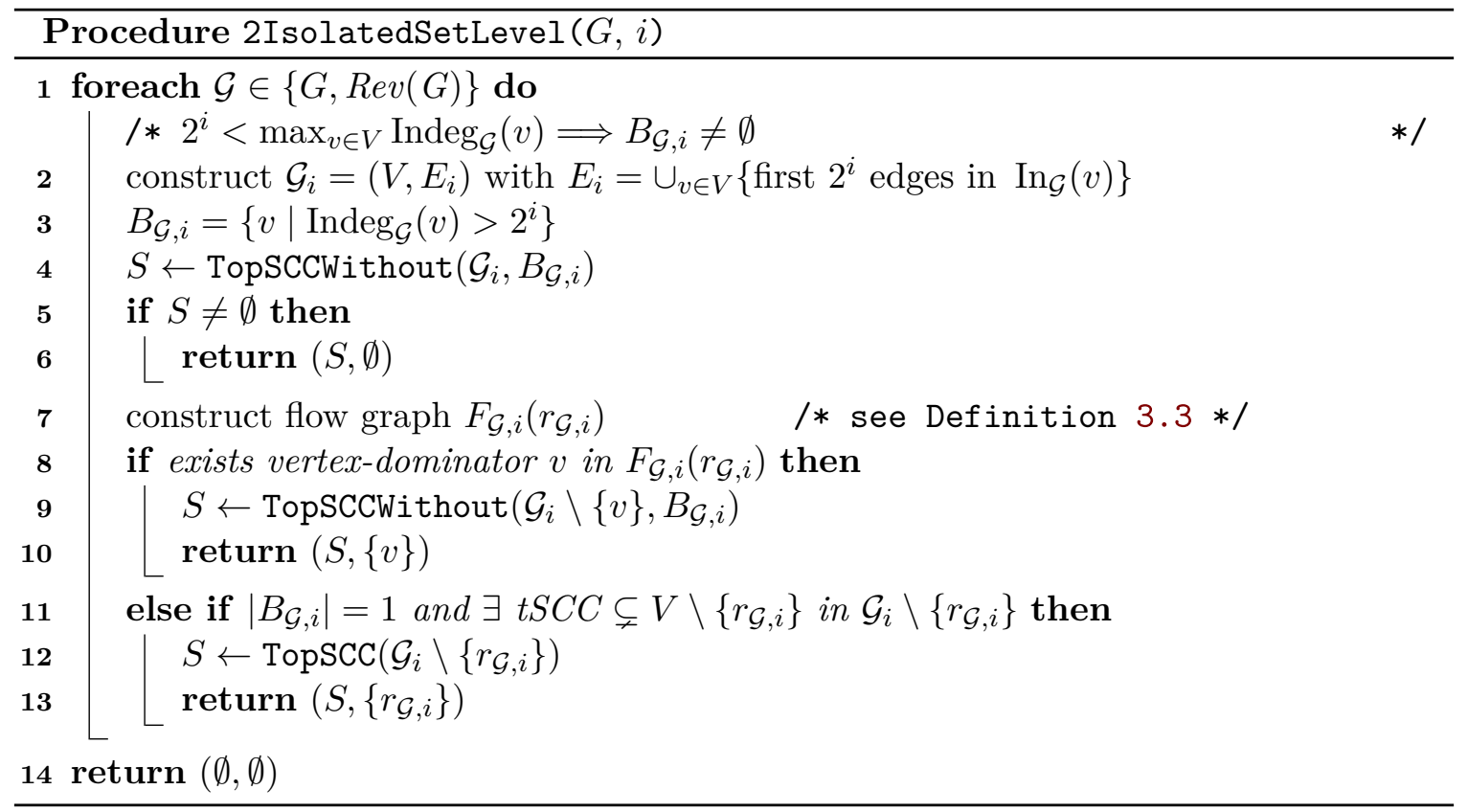

Let $\mathcal{G}_{i} \in\left\{G_{i}, \operatorname{Rev}(G)_{i}\right\}$. The Procedure 2IsolatedSetLevel first searches for a tSCC in $\mathcal{G}_{i}$ that does not contain a vertex of $B_{\mathcal{G}, i}$. If no such tSCC is found, the flow graph $F_{\mathcal{G}, i}\left(r_{\mathcal{G}, i}\right)$ is constructed and searched for vertex-dominators. If a vertex-dominator $v$ is found, a tSCC in $\mathcal{G}_{i} \backslash\{v\}$ that does not contain a vertex of $B_{\mathcal{G}, i}$ is found; one can show that such a tSCC always exists. We additionally have to consider the special case when $\left|B_{\mathcal{G}, i}\right|=1$. In this case we have $B_{\mathcal{G}, i}=\left\{r_{\mathcal{G}, i}\right\}$ and we want to detect when there exists an almost tSCC $\mathcal{G}[T]$ induced by some set of vertices $T$ with respect to $r_{\mathcal{G}, i}$ in $\mathcal{G}_{i}$ such that $V \backslash\left(T \cup\left\{r_{\mathcal{G}, i}\right\}\right)$ is not empty. We use Procedure TopSCCWithout $(H, B)$ to denote the search for a tSCC induced by vertices $S$ in a graph $H$ such that $S$ does not contain a vertex of $B$. Such a tSCC can simply be found by marking tSCCs in a standard SCC algorithm. We let all procedures that search for an SCC return the set of vertices $S$ in the SCC instead of the subgraph $G[S]$.

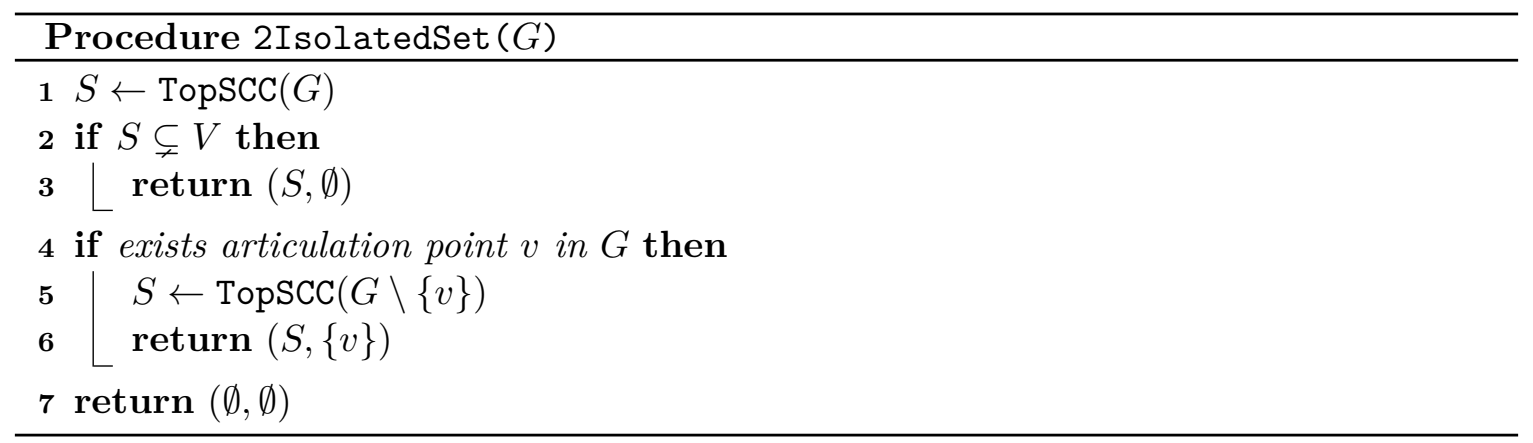

If no call to Procedure 2IsolatedSetLevel could identify an (almost) top or bottom SCC, we check in Procedure 2IsolatedSet whether the graph is strongly connected and 
either make progress by separating strongly connected components from each other or by finding an articulation point in the strongly connected graph. If an articulation point $v$ is found, disjoint top and bottom SCCs exist after the removal of the articulation point $v$. Procedure 2IsolatedSet returns a top SCC in $G \backslash\{v\}$ in this case. If the graph $G$ is strongly connected and does not contain an articulation point, then $G$ is a 2 vSCC. In this case the Procedure 2IsolatedSet returns the empty set, the recursion stops, and $2 \operatorname{vSCC}(G)$ returns G.

Whenever the algorithm identifies an (almost) top or bottom SCC induced by a set of vertices $S$, it recursively calls itself on $G[S \cup Z]$ and $G[V \backslash S]$ for $Z=\emptyset$ or $Z=\{v\}$, respectively. We use Lemma 3.2 to show that in this case every 2 vSCC of $G$ is completely contained in either $G[S \cup Z]$ or $G[V \backslash S]$, which will imply the correctness of the algorithm.

Theorem 4.3 (Correctness). Let $G$ be a simple directed graph. $2 \operatorname{vSCC}(G)$ computes the $2 v S C C s$ of $G$.

By stopping the recursion when the number of vertices is a small constant and distinguishing between the number of vertices $n^{\prime}$ at the current level of the recursion and the total number of vertices $n$, we can show that the runtime of $O\left(n^{\prime} \cdot \min \{|S|,|V \backslash S|\}\right)$ without recursion leads to a total runtime of $O\left(n^{2}\right)$.

Theorem 4.4 (Runtime). Procedure 2vSCC can be implemented in time $O\left(n^{2}\right)$.

\section{Extension to $\mathrm{kSCCs}$}

For any integral constant $k>2$ the presented algorithm extends to computing the $k$-edge and the $k$-vertex strongly connected components. In this section we outline the necessary changes, the details including all proofs are given in Appendix A.

Let an element of a graph $G$ denote an edge when keSCCs are searched for and a vertex when kvSCCs are searched for. We first extend the concepts of bridges, articulation points, and dominators from a single element to sets of elements with size less than $k$. A separator w.r.t. $k$-connectivity ( $k$-separator) is a minimal set of elements such that the set contains less than $k$ elements and its removal from the graph increases the number of SCCs in the graph. Two distinct vertices $u$ and $v$ are $k$-(strongly-) connected if they are strongly connected and they remain strongly connected after the removal of any less than $k$ elements different from $u$ and $v$ from $G$. The $k$-strongly connected components (kSCCs) of a graph $G$ are its maximal subgraphs $G[S]$ such that every pair of distinct vertices $u$ and $v$ in $S$ is $k$-connected in $G[S]$.For vertex-connectivity this definition allows for degenerate kvSCCs with $k$ or less vertices. Given the kvSCCs, the degenerate kvSCCs can be identified in linear time.

In a flow graph $G(r)$ a dominator $Z$ w.r.t. $k$-connectivity ( $k$-dominator) is a minimal set of less than $k$ elements in $G(r) \backslash\{r\}$ such that there exists a vertex $u \in G(r) \backslash(\{r\} \cup Z)$ such that $u$ is reachable from $r$ and every path from $r$ to $u$ contains an element of $Z$. We say that $Z k$-dominates $u$ in $G(r)$. Note that, in contrast to $k$-separators, the removal of a $k$-dominator from $G$ might not increase the number of SCCs but instead remove edges between SCCs. A $k$-dominator in a flow graph $G(r)$ and a $k$-separator in a graph $G$ can for edge-connectivity be found in time $O(m \log n)$ [Gab95] and for vertex-connectivity in time $O(m n)$ [Eve75, Gal80, HRG00, Gab06]. 
A set of vertices $T$ induces an almost $t S C C$ w.r.t. $k$-connectivity (k-almost $t S C C$ ) in $G$ with respect to a set of elements $Z$ with $|Z|<k$ if $G[T]$ is a tSCC in $G \backslash Z$ but has, for vertex-connectivity, incoming edges from each of the vertices in $Z$, or, for edge-connectivity, all the edges in $Z$ as incoming edges in $G$.

We adapt our algorithm as follows. For edge-connectivity we use different flow graphs: (1) We contract all vertices in $B_{G, i}$ to a single vertex, while keeping all edges between the vertices in $B_{G, i}$ and the remaining vertices as parallel edges. (2) We take the new contracted vertex as the root of the flow graph. With these definitions it is rather straightforward to extend the algorithm to keSCCs.

The extension to $k>2$ is more complicated for vertex-connectivity. In particular, we have to deal with the case $0<\left|B_{G, i}\right|<k$. Note that in this case we cannot use an additional vertex that we connect to the vertices of $B_{G, i}$ as the root in the flow graph because the vertices of $B_{G, i}$ would be a $k$-dominator in this flow graph independent of the underlying graph $G$. To be able to identify a set $Z \cap B_{G, i} \neq \emptyset$ with $|Z|<k$ for which a $k$-almost tSCC exists in $G$, we use $\left|B_{G, i}\right|<k$ different flow graphs. If the search in the $\left|B_{G, i}\right|$ flow graphs is not successful, we additionally search for a $\left(k-\left|B_{G, i}\right|\right)$-separator in $G_{i} \backslash B_{G, i}$ to detect the case $Z \supseteq B_{G, i}$. If such a $\left(k-\left|B_{G, i}\right|\right)$-separator $Z^{\prime}$ exists, then $Z=Z^{\prime} \cup B_{G, i}$ contains less than $k$ vertices and there exists a $k$-almost tSCC induced by some set of vertices $T \subsetneq V \backslash Z$ in $G_{i}$. These changes give the following result.

Theorem 5.1. For any integral constant $k>2 k e S C C s$ can be computed in time $O\left(n^{2} \log n\right)$ and kvSCCs in time $O\left(n^{3}\right)$. 2eSCCs can be computed in time $O\left(n^{2}\right)$.

\section{Acknowledgements}

We would like to thank Giuseppe Italiano for suggesting the problem and Slobodan Mitrović for helpful discussions. V. L. would like to thank Christian Tschabuschnig for his help in improving the readability of the algorithms.

\section{References}

[BGK $\left.{ }^{+} 08\right]$ Adam L. Buchsbaum, Loukas Georgiadis, Haim Kaplan, Anne Rogers, Robert E. Tarjan, and Jeffery R. Westbrook. "Linear-Time Algorithms for Dominators and Other Path-Evaluation Problems". In: SIAM Journal on Computing 38.4 (2008). Announced at STOC'98 and SODA'04, pp. 1533-1573 (cit. on pp. 2, 3, $5)$.

[BJG09] Jørgen Bang-Jensen and Gregory Gutin. Digraphs: Theory, Algorithms and Applications. 2nd ed. Springer Monographs in Mathematics. London: Springer, 2009 (cit. on p. 1).

[BM76] John A. Bondy and U. S. R. Murty. Graph Theory with Applications. London: Macmillan, 1976 (cit. on p. 1).

[CH14] Krishnendu Chatterjee and Monika Henzinger. "Efficient and Dynamic Algorithms for Alternating Büchi Games and Maximal End-component Decomposition". In: Journal of the ACM 61.3 (2014). Announced at SODA'11 and SODA'12, 15:1-15:40 (cit. on p. 2). 
[CHL15] Krishnendu Chatterjee, Monika Henzinger, and Veronika Loitzenbauer. "Improved Algorithms for One-Pair and k-Pair Streett Objectives". In: Symposium on Logic in Computer Science (LICS). to appear. 2015 (cit. on p. 2).

[CJH03] Krishnendu Chatterjee, Marcin Jurdziński, and Thomas A. Henzinger. "Simple Stochastic Parity Games". In: Annual Conference on Computer Science Logic (CSL). Springer, 2003, pp. 100-113 (cit. on pp. 3, 29).

[EGI ${ }^{+}$97] David Eppstein, Zvi Galil, Giuseppe F. Italiano, and Amnon Nissenzweig. "Sparsification-A Technique for Speeding Up Dynamic Graph Algorithms". In: Journal of the ACM 44.5 (Sept. 1997). Announced at FOCS'92, pp. 669-696 (cit. on p. 3).

[Eve75] Shimon Even. "An Algorithm for Determining Whether the Connectivity of a Graph is at Least k". In: SIAM Journal on Computing 4.3 (1975), pp. 393-396 (cit. on pp. 2, 10).

[Gab06] Harold N. Gabow. "Using Expander Graphs to Find Vertex Connectivity". In: Journal of the ACM 53.5 (2006). Announced at FOCS'00, pp. 800-844 (cit. on pp. 2, 10).

[Gab95] Harold N. Gabow. "A Matroid Approach to Finding Edge Connectivity and Packing Arborescences". In: Journal of Computer and System Sciences 50.2 (1995). Announced at STOC'91, pp. 259-273 (cit. on p. 10).

[Gal80] Zvi Galil. "Finding the Vertex Connectivity of Graphs". In: SIAM Journal on Computing 9.1 (1980), pp. 197-199 (cit. on pp. 2, 10).

[Geo10] Loukas Georgiadis. "Testing 2-Vertex Connectivity and Computing Pairs of Vertex-Disjoint $s$ - $t$ Paths in Digraphs". In: ICALP 2010. 2010, pp. 433-442 (cit. on p. 2).

[GI91] Zvi Galil and Giuseppe F. Italiano. "Reducing Edge Connectivity to Vertex Connectivity". In: ACM SIGACT News 22.1 (1991), pp. 57-61 (cit. on p. 2).

[GIL $\left.{ }^{+} 15 \mathrm{a}\right]$ Loukas Georgiadis, Giuseppe F. Italiano, Luigi Laura, and Nikos Parotsidis. "2-Edge Connectivity in Directed Graphs". In: SODA. 2015, pp. 1988-2005 (cit. on pp. 2-5).

[GIL ${ }^{+}$15b] Loukas Georgiadis, Giuseppe F. Italiano, Luigi Laura, and Nikos Parotsidis. "2-Vertex Connectivity in Directed Graphs". In: ICALP. to appear. 2015 (cit. on pp. 2, 5).

[GT85] Harold N. Gabow and Robert Endre Tarjan. "A Linear-Time Algorithm for a Special Case of Disjoint Set Union". In: Journal of Computer and System Sciences 30.2 (1985). Announced at STOC'83, pp. 209-221 (cit. on pp. 2, 3).

[HKW99] Monika Henzinger, Valerie King, and Tandy Warnow. "Constructing a Tree from Homeomorphic Subtrees, with Applications to Computational Evolutionary Biology". In: Algorithmica 24.1 (1999). Announced at SODA'96, pp. 1-13 (cit. on p. 2).

[HRG00] Monika R. Henzinger, Satish Rao, and Harold N. Gabow. "Computing Vertex Connectivity: New Bounds from Old Techniques". In: Journal of Algorithms 34.2 (2000). Announced at FOCS'96, pp. 222-250 (cit. on pp. 2, 10). 
[HT73] John E. Hopcroft and Robert Endre Tarjan. "Dividing a Graph into Triconnected Components". In: SIAM Journal on Computing 2.3 (1973), pp. 135-158 (cit. on p. 2).

[ILS12] Giuseppe F. Italiano, Luigi Laura, and Federico Santaroni. "Finding strong bridges and strong articulation points in linear time". In: Theoretical Computer Science 447 (2012). Announced at COCOA'10, pp. 74-84 (cit. on pp. 2, 4, 6).

[Jab14] Raed Jaberi. "On computing the 2-vertex-connected components of directed graphs". 2014 (cit. on pp. 2, 5).

[KMW98] Sanjeev Khanna, Rajeev Motwani, and Randall H. Wilson. "On Certificates and Lookahead in Dynamic Graph Problems". In: Algorithmica 21.4 (1998). Announced at SODA'96, pp. 377-394 (cit. on p. 3).

[Mak88] Seishi Makino. "An algorithm for finding all the $k$-components of a digraph". In: International Journal of Computer Mathematics 24.3-4 (1988), pp. 213-221 (cit. on p. 2).

[New10] Mark E. J. Newman. Networks: An Introduction. Oxford University Press, 2010 (cit. on p. 1).

[NI08] Hiroshi Nagamochi and Toshihide Ibaraki. Algorithmic Aspects of Graph Connectivity. New York: Cambridge University Press, 2008 (cit. on p. 1).

[NI92] Hiroshi Nagamochi and Toshihide Ibaraki. "A Linear-Time Algorithm for Finding a Sparse $k$-Connected Spanning Subgraph of a $k$-Connected Graph". In: Algorithmica 7.5\&6 (1992), pp. 583-596 (cit. on p. 2).

[NW93] Hiroshi Nagamochi and Toshimasa Watanabe. "Computing $k$-Edge-Connected Components of a Multigraph". In: IEICE Transactions on Fundamentals of Electronics, Communications and Computer Sciences E76-A.4 (1993), pp. 513517 (cit. on p. 2).

[Tar72] Robert E. Tarjan. "Depth-First Search and Linear Graph Algorithms". In: SIAM Journal on Computing 1.2 (1972). Announced at SWAT'71 (FOCS), pp. 146-160 (cit. on pp. 2-4).

[Tar76] Robert E. Tarjan. "Edge-disjoint spanning trees and depth-first search". In: Acta Informatica 6.2 (1976), pp. 171-185 (cit. on pp. 2, 3). 


\section{Appendix}

\section{A Missing proofs and extension to $\mathrm{kSCCs}$}

In this section we describe the algorithms for $k$-edge and $k$-vertex strongly connected components and prove their correctness and running time. This in particular implies the results presented in the main part of the paper. We use the definitions of Sections 2 and 5 , otherwise the section is self-contained. We analyze most parts simultaneously for edge and vertex connectivity but explicitly point out important differences. Note that the text is meant to be read consistently for either edge or vertex connectivity, e.g., when a statement about $k$-connectivity is interpreted as a statement about $k$-vertex connectivity, then any occurrence of "element" has to be interpreted as a vertex and not as an edge.

\section{A.1 New top SCCs and $k$-dominators in subgraphs}

We introduce the notion of an isolated set with respect to $k$-connectivity, $k$-isolated set for short, for the informal introduction of our approach. Let for edge connectivity a $k$-isolated set $S \subsetneq V$ be a set of vertices with less than $k$ incoming edges and let $U=V \backslash S$. Recall that we use "incoming edges of a set of vertices $S$ " to denote the edges from $V \backslash S$ to $S$. For vertex connectivity, let a $k$-isolated set $S$ be a set of vertices with (1) incoming edges from less than $k$ vertices and for which (2) the set of vertices $U \subseteq V \backslash S$ that have no edges to vertices of $S$ is not empty. Clearly, for both edge and vertex connectivity, no element of $S$ is $k$-connected to any vertex of $U$ because there cannot exist $k$ disjoint paths from any vertex of $U$ to any vertex of $S$. the graph is strongly connected and does not contain a $k$-separator, i.e., the graph is $k$-connected. Think of the following simple recursive algorithm to output the kSCCs of graph $G$ :

1. Find a $k$-isolated set $S$ in $G$.

2. If none exists, output $G$.

3. Otherwise recurse on the graphs induced by $S$ and $U$; for vertex connectivity add the vertices in $V \backslash(S \cup U)$ to both $S$ and $U$ before the recursion.

Our algorithms follow this scheme for specific $k$-isolated sets. As the recursion depth can be $\Theta(n)$, to achieve an $o(m n)$ running time, we cannot afford to look at all edges in each level of recursion. Thus our algorithms are based on the following question:

Can we identify $k$-isolated sets by searching in a proper subgraph of $G$ ?

We first explain the $k$-isolated sets our algorithms identify, formalize the correctness idea outlined above for this kind of $k$-isolated sets, and provide an intuition why and when they can be identified in proper subgraphs of $G$. We then formalize the latter in the remaining part of this subsection. The results in this subsection are formulated for general subgraphs such that they can also be used for the $O\left(\mathrm{~m}^{2} / \log n\right)$-algorithm for 2eSCCs presented in Appendix B. Our algorithms for kSCCs are described in Subsection A.2.

The $k$-isolated sets we identify are tSCCs and $k$-almost tSCCs. Recall the definition of $k$-almost tSCCs: A set of vertices $T$ induces a $k$-almost $t S C C$ in $G$ with respect to a set of elements $Z$ with $|Z|<k$ if $G[T]$ is a tSCC in $G \backslash Z$ but has, for vertex connectivity, incoming edges from each of the vertices in $Z$, or, for edge connectivity, all the edges in $Z$ as incoming edges in $G$. For the sake of a compact formulation, we introduce the notation 
$k$-almostTSCC $(T, Z, G)$ for a $k$-almost tSCC in $G$ with respect to a set of elements $Z$ with $|Z|<k$ induced by the set of vertices $T$. For edge connectivity each $k$-almost tSCC is induced by a $k$-isolated set. For vertex connectivity this holds whenever there exist vertices without edges to the $k$-almost tSCC; our algorithm only identifies $k$-almost tSCCs that are induced by $k$-isolated sets.

For the intuition behind the definition of $k$-almost tSCCs, think of a strongly connected graph that contains a $k$-separator $Z$ (see Section 5 ). Recall that we require $k$-separators to be minimal (with respect to set inclusion), i.e., $Z$ is a minimal set of less than $k$ elements such that $G \backslash Z$ is not strongly connected. For a $k$-separator $Z$ there exist both a tSCC and a bSCC in $G \backslash Z$ that were adjacent to $Z$ in $G$ and are disjoint. Let $T$ be the vertices in a tSCC in $G \backslash Z$ (there can be more than one for vertex connectivity). Observe that $T$ is a $k$-isolated set in $G$ and induces a $k$-almostTSCC $(T, Z, G)$. Further, tSCCs can be identified in time linear in the number of edges in $G$ by a standard SCC algorithm by simply marking the SCCs without incoming edges. To see why the notion of $k$-isolated sets is helpful when searching in a subgraph of $G$, note the following: If $T$ contains only a few vertices, then each vertex in $T$ has low in-degree in $G$ because all incoming edges of a vertex of $T$ in $G$ either come from other vertices in $T$ or, for edge connectivity, are the edges in $Z$, and, for vertex connectivity, come from the vertices in $Z$. In our algorithms for kSCCs we search for tSCCs and $k$-almost tSCCs in the subgraph of $G$ induced by vertices with low in-degree. We do the same on $\operatorname{Rev}(G)$ to detect small bSCCs and small $k$-almost bSCCs (defined analogously).

To identify a $k$-almostTSCC $(T, Z, G)$, we do not only have to find the tSCC $G[T]$ in $G \backslash Z$ but first have to identify the set of elements $Z$. Assume there exists a vertex $r \notin Z$ that is not in $T$ but can reach all elements in $Z$. Since $G[T]$ is a tSCC in $G \backslash Z$, it follows that $Z k$-dominates every vertex of $T$ in the flow graph $G(r)$. We formalize this observation and the intuition about the correctness of our approach of repeatedly identifying tSCCs and $k$-almost tSCCs (that are also $k$-isolated sets) in the following lemma.

Lemma A.1 (Extension of Lemma 3.2 to $k$-connectivity). Let $T$ and $Z$ be such that a $k$-almostTSCC $(T, Z, G)$ exists. Let $W=V \backslash T$ for edge connectivity and let $W=V \backslash(T \cup Z)$ for vertex connectivity. Assume $W \neq \emptyset$. Then no vertex of $W$ is $k$-connected to any vertex of $T$. Additionally, the set $Z \cap G(r)$ is a $k$-dominator in $G(r)$ for every $r \in W$ for which $Z \cap G(r)$ is not empty.

Proof. By the definition of a tSCC, the vertices in $T$ are strongly connected in $G \backslash Z$ but have no incoming edges from vertices of $W$ in $G \backslash Z$. Hence in $G$ every path from a vertex of $W$ to a vertex of $T$ contains an element of $Z$. This implies that $Z \cap G(r)$ is a $k$-dominator in $G(r)$ for every $r \in W$. For vertex connectivity we have that the vertices in $W$ have no edges to the vertices in $T$ in $G$; hence no vertex of $W$ is $k$-connected to a vertex of $T$.

Let $r \in V$ be a vertex such that there does not exist a set of vertices $S$ with $r \notin S$ that induces a tSCC in $G$, i.e., all vertices in $V$ can be reached from $r$. We show below that this is a sufficient condition such that whenever $Z$ is a $k$-dominator in the flow graph $G(r)$, then there exists a set $T$ that induces a tSCC in $G \backslash Z$ and $k$-almostTSCC( $T, Z, G)$ indeed exists. Thus if we only want to detect tSCCs and $k$-almost tSCCs $k$-almostTSCC $(T, Z, G)$ for which both $T$ and $Z$ do not contain $r$, we can use the following approach to find one of them whenever one exists:

1. Search for a set of vertices $T$ with $r \notin T$ that induces a tSCC in $G$. 
2. If none is found, search for a $k$-dominator in $G(r)$.

3. If a $k$-dominator $Z$ is found, find a set of vertices $T$ with $r \notin T$ that induces a tSCC in $G \backslash Z$.

We formalize the correctness of this approach with the following two lemmata.

Lemma A.2. Let $r$ be a vertex that can reach all vertices in $G$ and let $G(r)$ be the flow graph rooted at $r$. Let $Z$ be a $k$-dominator in $G(r)$. Then there exists a set of vertices $T$ with $r \notin T$ that is a $t S C C$ in $G \backslash Z$.

Proof. Let $D$ be the vertices dominated by $Z$ in $G(r)$. Since $D$ is dominated by $Z$, there are no edges from vertices in $G(r) \backslash(D \cup Z)$ to vertices in $D$ in $G \backslash Z$. Thus either $D$ contains a tSCC that does not contain $r$ in $G \backslash Z$ or there are vertices in $G \backslash G(r)$ that have edges to vertices in $D$. By assumption $G \backslash G(r)$ is empty.

Lemma A.3. Let $G(r)$ be a flow graph for some graph $G=(V, E)$ and some vertex $r \in V$. Let $Z$ be a $k$-dominator in $G(r)$ and let the set of vertices $T$ with $r \notin T$ induce a $t S C C$ in $G \backslash Z$. Then k-almostTSCC $(T, Z, G)$ exists.

Proof. Since $T$ induces a tSCC in $G \backslash Z$, the incoming edges of $T$ are clearly a subset of $Z$ for edge connectivity and the edges from $Z$ to $T$ for vertex connectivity. It remains to show that $T$ has an incoming edge for each element of $Z$, i.e., that $Z$ satisfies the minimality condition for $k$-almost tSCCs. Assume by contradiction that $k$-almostTSCC $\left(T, Z^{\prime}, H\right)$ exists for a proper subset $Z^{\prime}$ of $Z$. Then $r$ can reach $T$ only through the elements of $Z^{\prime}$, i.e., $Z^{\prime}$ is a $k$-dominator in $G(r)$, a contradiction to the minimality of $k$-dominators.

Now we want to generalize this approach to subgraphs $H$ of $G$, that is, for some appropriately chosen root $r$, we first search for a tSCC in $H$ not containing $r$; if no tSCC is found, we search for a $k$-dominator in the flow graph $H(r)$; and for a $k$-dominator $Z$ in $H(r)$ we find a tSCC in $H \backslash Z$ not containing $r$. If no tSCC is found in $H$ but a $k$ dominator $Z$ is found, this yields a $k$-almostTSCC $(T, Z, H)$ by Lemma A.3. But when does a $k$-almostTSCC $(T, Z, H)$ imply a $k$-almostTSCC $(T, Z, G)$ ? First think of a tSCC in $H$ induced by a set of vertices $T$. We are only allowed to look at the edges in $H$ but assume for now we know for each vertex whether all its incoming edges in $G$ are also contained in $H$. If some vertex of $T$ has more incoming edges in $G$ than in $H$, we cannot decide whether $T$ also induces a tSCC in $G$. However, if for each vertex of $T$ all its incoming edges in $G$ are also in $H$, then $T$ induces a tSCC in $G$ as well. Now think of a $k$-almostTSCC( $T, Z, H)$. If the incoming edges of each vertex in $T$ are present in $H$, then also the elements of $Z$ have to be contained in $H$ because otherwise at least one vertex of $T$ would miss an incoming edge in $H$. Thus by the observation for tSCCs above we have that $k$-almostTSCC $(T, Z, G)$ exists. We state this formally for slightly more general graphs $H$ in the following lemma and its corollary such that we can apply these results to all graphs we define in this work. Consider a graph $H=\left(V_{H}, E_{H}\right)$ constructed from a graph $G=(V, E)$ that is not necessarily a subgraph of $G$ but has the following guarantee for all vertices in some set $A \subseteq V_{H} \cap V$ : For each vertex $u$ of $A$ the incoming edges of $u$ in $H$ are the same as in $G$.

Lemma A.4 (Extension of Lemma 3.4). Let $G=(V, E)$ and $H=\left(V_{H}, E_{H}\right)$ be two graphs with the following guarantee for all vertices in $A \subseteq V_{H} \cap V$ : For each vertex $u \in A$ we have $\operatorname{In}_{H}(u)=\operatorname{In}_{G}(u)$. Then a set of vertices $T \subseteq A$ induces a $t S C C$ in $H$ if and only if it induces a $t S C C$ in $G$. 
Proof. By the guarantee on $A$, the incoming edges of the vertices in $T$ are the same in $H$ and $G$, i.e., we have $H[T]=G[T]$. Furthermore, the set of vertices $T$ has no incoming edges in $H$ if and only if it has no incoming edges in $G$.

Corollary A.5 (Extension of Corollary 3.5). Let G, H, and $A$ be as in Lemma A.4. For a set of vertices $T \subseteq A$ and a set of elements $Z$ we have that $k$-almostTSCC $(T, Z, H)$ exists if and only if $k$-almostTSCC $(T, Z, G)$ exists.

Our algorithms identify tSCCs and $k$-almost tSCCs that are subsets of $A$ in a graph $H$ with the guarantee described above. Together with Lemma A.1 this is crucial for the correctness of our approach.

To obtain algorithms with a good running time, we want to identify a tSCC or a $k$-almost tSCC that is a subset of $A$ in $H$ whenever a tSCC or $k$-almost tSCC that is a subset of $A$ (and is a $k$-isolated set) exists in $G$. Note that we cannot say anything about tSCCs in $H$ that contain vertices that are not in $A$. Recall that we find $k$-almost tSCCs by searching for $k$-dominators $Z$ in flow graphs of $H$ and then searching for tSCCs in $H \backslash Z$. Further, recall that since we want to achieve a running time of $o(m n)$ (at least for edge connectivity or for $k=2$ ), we cannot afford to recompute all strongly connected components and thus cannot assume that the graph the algorithm currently operates on is strongly connected. Hence to argue about which $k$-almost tSCCs in $G$ we can identify by the search in a flow graph of $H$, we have to consider the reachability of a set $Z$ for which a $k$-almostTSCC $(T, Z, G)$ exists from the root of the flow graph in $H$. The flow graphs used by our algorithms contain all vertices in $V_{H} \backslash A$. Thus whenever some elements of a set $Z$ are not reachable from the root of such a flow graph, i.e., are not in $H(r)$, then there exists a non-empty subset of $A$ that is not reachable from vertices in $V_{H} \backslash A$. Thus in this case there exists a tSCC in $H$ that contains only vertices in $A$ and is also a tSCC in $G$ by Lemma A.4. Hence in this case our algorithms can make progress by identifying in $H$ a tSCC that only contains vertices in $A$ instead of searching for the $k$-almost tSCC with respect to $Z$.

Observation A.6. Let $G=(V, E), H=\left(V_{H}, E_{H}\right)$, and $A$ be as in Lemma A.4. Let $H(r)$ be a flow graph in $H$ for some root $r \in V_{H}$ that contains all vertices in $V_{H} \backslash A$. If some vertices of $A$ are not contained in $G(r)$, then there exists a set of vertices $S \subseteq A$ that induces a $t S C C H[S]$ in $H$.

We now come back to proper subgraphs of graphs. Let $G_{h}=\left(V_{h}, E_{h}\right)$ be a subgraph of a graph $G=(V, E)$, i.e., $V_{h} \subseteq V$ and $E_{h} \subseteq G\left[V_{h}\right]$. We use the index $h$ to identify specific subgraphs and the corresponding sets of edges and vertices. Let $A_{G, h}$ be the set of vertices in $G_{h}$ for which we can guarantee for each $u \in A_{G, h}$ that $\operatorname{In}_{G_{h}}(u)=\operatorname{In}_{G}(u)$ and let $B_{G, h}$ denote $V_{h} \backslash A_{G, h}$ (which is a subset of $V \backslash A_{G, h}$ ). If $B_{G, h}=\emptyset$, then $G_{h}=G$ and we can simply search for SCCs and $k$-separators in $G$ to make progress in our algorithms. Thus we assume $B_{G, h} \neq \emptyset$ in the remainder of this subsection. We next define graphs and flow graphs derived from $G_{h}$ in which we can identify a set $Z$ as a $k$-dominator in the flow graph whenever a $k$-almostTSCC $(T, Z, G)$ with $T \subseteq A_{G, h}$ exists and each element of $Z$ is reachable from the root of the flow graph - except for one special case for vertex connectivity, which we consider separately. Intuitively, in the graphs derived from $G_{h}$ we view the vertices in $B_{G, h}$ as a (directed) clique. Since we do not know anything about the structure of the subgraph induced by $B_{G, h}$, this is a "worst-case" assumption on the connectivity of these vertices with respect to detecting $k$-isolated sets. 
We first define the graphs and flow graphs derived from $G_{h}$ for edge connectivity, then for vertex connectivity. For edge connectivity we are able to identify a $k$-almostTSCC $(T, Z, G)$ with $T \subseteq A_{G, h}$ for which the edges in $Z$ are reachable from the vertices in $B_{G, h}$ by using the following way of contracting the vertices in $B_{G, h}$ and use the new contracted vertex as the root in the flow graph.

Definition A.7 (Flow graph for edge connectivity). Let $G_{h}=\left(V_{h}, E_{h}\right)$ be a subgraph of a graph $G=(V, E)$. Let $A_{G, h}$ be the set of vertices in $G_{h}$ for which we can guarantee for each $u \in A_{G, h}$ that $\operatorname{In}_{G_{h}}(u)=\operatorname{In}_{G}(u)$ and let $B_{G, h}$ denote $V_{h} \backslash A_{G, h}$.

For edge connectivity and $\left|B_{G, h}\right| \geq 1$, we define the flow graph $F_{G, h}\left(r_{G, h}\right)$ as follows. Let the graph $F_{G, h}$ be the multi-graph $F_{G, h}=\left(V_{h}^{\prime}, E_{h}^{\prime}\right)$ where all vertices in $B_{G, h}$ are contracted to a single vertex $r_{G, h}$ in the following way. The vertices $V_{h}^{\prime}$ are equal to $A_{G, h} \cup\left\{r_{G, h}\right\}$ and the edges $E_{h}^{\prime}$ consists of all edges in $G\left[A_{G, h}\right]$ and one edge $\left(u, r_{G, h}\right)$ for each edge in $(u, v) \in$ $E \cap\left(A_{G, h} \times B_{G, h}\right)$ and, symmetrically, one edge $\left(r_{G, h}, v\right)$ for each $(u, v) \in E \cap\left(B_{G, h} \times A_{G, h}\right)$.

With the flow graph $F_{G, h}\left(r_{G, h}\right)$ we have the following strategy to identify a tSCC or a $k$-almost tSCC in $G_{h}$ and $F_{G, h}$ whenever a tSCC or a $k$-almost tSCC that is a subset of $A_{G, h}$ exists in $G$ :

1. Search for a tSCC induced by vertices in $A_{G, h}$ in $G_{h}$.

2. If none found, search for a $k$-dominator in $F_{G, h}\left(r_{G, h}\right)$.

3. If a $k$-dominator $Z$ is found, find a tSCC induced by vertices in $A_{G, h}$ in $G_{h} \backslash Z$.

We detect a tSCC in the first step whenever some vertex of $A_{G, h}$ is not reachable from any vertex of $B_{G, h}$. Thus if no such tSCC exists, all vertices of $A_{G, h}$ are contained in $F_{G, h}\left(r_{G, h}\right)$. This has two consequences. First, if a $k$-dominator $Z$ is found in the second step, by Lemma A.3 a tSCC induced by vertices $T \subseteq A_{G, h}$ exists in $G_{h} \backslash Z$. In this case $k$-almostTSCC $(T, Z, H)$ exists by Lemma A.3 and $k$-almostTSCC $(T, Z, G)$ exists by Corollary A.5; this is crucial for the correctness of the approach. Second, if for some set of vertices $T \subseteq A_{G, h}$ and some set of edges $Z$ a $k$-almostTSCC $(T, Z, G)$ exists, then $Z$ is contained in $F_{G, h}\left(r_{G, h}\right)$; thus in this case a $k$-dominator is identified in $F_{G, h}\left(r_{G, h}\right)$.

For vertex connectivity we have to use different flow graphs because we also want to identify a $k$-almostTSCC $(T, Z, G)$ with $T \subseteq A_{G, h}$ for which some of the vertices in $Z$ are contained in $B_{G, h}$. We first consider the simpler case when $\left|B_{G, h}\right| \geq k$. In this case we connect an artificial root vertex to all vertices in $B_{G, h}$, which allows us to detect a set $Z$ for which a $k$-almostTSCC $(T, Z, G)$ exists when each vertex in $Z$ is either contained in $B_{G, h}$ or reachable from a vertex in $B_{G, h}$. When $0<\left|B_{G, h}\right|<k$ we cannot use an additional vertex that we connect to the vertices of $B_{G, h}$ as root in the flow graph because the vertices of $B_{G, h}$ would be a $k$-dominator in this flow graph independent of the underlying graph $G$. We still want to detect a $k$-almostTSCC $(T, Z, G)$ with $T \subseteq A_{G, h}$ for which $Z$ includes vertices in $B_{G, h}$ in this case. We further distinguish two cases: Either there exists a vertex in $B_{G, h}$ that is not in $Z$ or $B_{G, h}$ is contained in $Z$. For the first case we use $\left|B_{G, h}\right|<k$ different flow graphs and we can identify the set of elements in $Z$ a flow graph for a vertex in $B_{G, h} \backslash Z$ when each vertex in $Z$ is either contained in $B_{G, h}$ or reachable from a vertex in $B_{G, h}$. In the second case, i.e., $B \subseteq Z$, we cannot identify $Z$ in any of the flow graphs; we consider this case explicitly by testing whether $G_{h} \backslash B_{G, h}$ is strongly connected and searching for a $\left(k-\left|B_{G, h}\right|\right)$-separator in $G_{h} \backslash B_{G, h}$. 


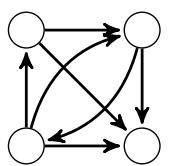

$G$

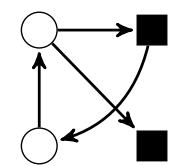

$G_{h}$

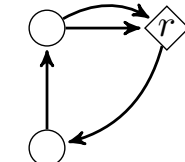

$F_{G, h}$ for edge $F_{G, h}$ for vertex

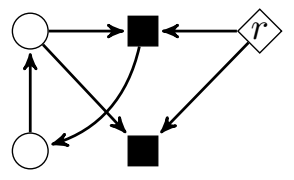

$F_{G, h, w}$

Figure 1: Simple example graphs for graphs and vertices as in Definitions A.7 and A.8. The black square vertices denote vertices of $B_{G, h}$, the white diamond vertices denote the vertices that are used as roots in the corresponding flow graphs.

Definition A.8 (Flow graphs for vertex connectivity). Let $G_{h}=\left(V_{h}, E_{h}\right)$ be a subgraph of a graph $G=(V, E)$. Let $A_{G, h}$ be the set of vertices in $G_{h}$ for which we can guarantee for each $u \in A_{G, h}$ that $\operatorname{In}_{G_{h}}(u)=\operatorname{In}_{G}(u)$ and let $B_{G, h}$ denote $V_{h} \backslash A_{G, h}$.

- For vertex connectivity and $\left|B_{G, h}\right| \geq k$, we define the flow graph $F_{G, h}\left(r_{G, h}\right)$ as follows. Let $F_{G, h}$ be the graph $G_{h}$ with an additional vertex $r_{G, h}$ and an additional edge from $r_{G, h}$ to each vertex in $B_{G, h}$.

- For vertex connectivity and $0<\left|B_{G, h}\right|<k$, we define $\left|B_{G, h}\right|$ different flow graphs, one for each $w \in B_{G, h}$. Let $F_{G, h, w}\left(r_{G, h, w}\right)$ denote the flow graph for $w \in B_{G, h}$. The root $r_{G, h, w}$ is equal to $w$ and the graph $F_{G, h, w}$ is the graph $G_{h}$ with an additional edge from $w$ to each vertex in $B_{G, h} \backslash\{w\}$.

The approach for vertex connectivity differs from the approach for edge connectivity mainly by (1) a different definition of the flow graph $F_{G, h}\left(r_{G, h}\right)(2)$ in the case $0<\left|B_{G, h}\right|<k$ by (2a) searching in $\left|B_{G, h}\right|$ different flow graphs and (2b) considering the special case to detect when a $k$-almostTSCC $(T, Z, G)$ with $Z \supseteq B_{G, h}$ exists. We use the following lemma to show the correctness for the special case.

Lemma A.9. Consider vertex connectivity and assume $0<\left|B_{G, h}\right|<k$. Assume that no tSCC induced by vertices in $A_{G, h}$ exists in $G_{h}$ and that no $k$-dominator exists in any of the flow graphs $F_{G, h, w}\left(r_{G, h, w}\right)$ for $w \in B_{G, h}$.

(a) If $G_{h} \backslash B_{G, h}$ is not strongly connected, let $T$ be a set of vertices that induces a $t S C C$ in $G_{h} \backslash B_{G, h}$ and let $Z^{\prime}=\emptyset$.

(b) If $G_{h} \backslash B_{G, h}$ is strongly connected, $\left|B_{G, h}\right|<k-1$, and there exists a $\left(k-\left|B_{G, h}\right|\right)$ separator $Z^{\prime}$ in $G_{h} \backslash B_{G, h}$, let $T$ be a set of vertices that induces a $t S C C$ in $G_{h} \backslash\left(Z^{\prime} \cup B_{G, h}\right)$. Let $Z=Z^{\prime} \cup B_{G, h}$. If either the conditions for $(a)$ or for $(b)$ hold, then $k$-almostTSCC $(T, Z, G)$ exists.

Proof. First note that $T \subseteq A_{G, h}$, that there are edges from $V \backslash T$ to $T$ in $G_{h}$, and that these edges are all from vertices of $Z$. Thus it remains to show that each vertex of $Z$ has an edge to a vertex of $T$. In Case (b) the existence of a vertex in $Z^{\prime}$ that has no edge to $T$ would contradict the minimality of a $\left(k-\left|B_{G, h}\right|\right)$-separator (thus $k$-almostTSCC $\left(T, Z^{\prime}, G_{h} \backslash B_{G, h}\right)$ exists). To see that also each vertex of $B_{G, h}$ has an edge to a vertex of $T$, assume by contradiction that there exists a set of vertices $U \subseteq B_{G, h}$ such that there is no edge from $U$ to $T$. By the assumption that no tSCC induced by vertices in $A_{G, h}$ exists in $G_{h}$, we have that all vertices in $A_{G, h}$ are reachable from some vertex of $B_{G, h}$ and thus contained 
in $F_{G, h, w}\left(r_{G, h, w}\right)$ for all $w \in B_{G, h}$. Hence $Z \backslash U$ would dominate the vertices in $T$ in $F_{G, h, u}\left(r_{G, h, u}\right)$ for $u \in U$, a contradiction to our assumptions.

The following two corollaries together with Lemmata A.1, A.4, and A.9 provide a summary of the results in this subsection.

Corollary A.10. Assume $B_{G, h}$ is such that the flow graph $F_{G, h}\left(r_{G, h}\right)$ is defined. If no $t S C C$ induced by vertices in $A_{G, h}$ exists in $G_{h}$ and a set of elements $Z$ with $|Z|<k$ is a $k$-dominator in $F_{G, h}\left(r_{G, h}\right)$, then for some set of vertices $T \subseteq A_{G, h} k$-almostTSCC $(T, Z, G)$ exists. The same holds for the flow graphs $F_{G, h, w}\left(r_{G, h, w}\right)$ for $w \in B_{G, h}$ whenever they are defined.

Proof. Since no tSCC induced by vertices in $A_{G, h}$ exists in $G_{h}$, all vertices in $A_{G, h}$ are reachable from some vertex of $B_{G, h}$ in $G_{h}$ and thus contained in $F_{G, h}\left(r_{G, h}\right)$ (or for vertex connectivity and $\left|B_{G, h}\right|<k$ in $F_{G, h, w}\left(r_{G, h, w}\right)$ for all $\left.w \in B_{G, h}\right)$. Thus there exists a tSCC induced by a set of vertices $T \subseteq A_{G, h}$ in $F_{G, h} \backslash Z$ (Lemma A.2) and thus also in $G_{h} \backslash Z$ (Lemma A.4). By Lemma A.3 this implies that $k$-almostTSCC $\left(T, Z, G_{h}\right)$ exists. Thus by Corollary A.5 $k$-almostTSCC $(T, Z, G)$ exists.

Corollary A.11 (Extension of Lemma 3.6). Assume $B_{G, h}$ is such that the flow graph $F_{G, h}\left(r_{G, h}\right)$ is defined. Let $T \subseteq A_{G, h}$. If no $t S C C$ induced by vertices in $A_{G, h}$ exists in $G$, for some set of elements $Z$ with $|Z|<k$-almostTSCC $(T, Z, G)$ exists, and $B_{G, h} \backslash Z$ is not empty, then $Z$ is a $k$-dominator in $F_{G, h}\left(r_{G, h}\right)$. The same holds for at least one of the flow graphs $F_{G, h, w}\left(r_{G, h, w}\right)$ for $w \in B_{G, h}$ whenever they are defined.

Proof. By Corollary A.5 $k$-almostTSCC $\left(T, Z, G_{h}\right)$ exists. Since no tSCC induced by vertices in $A_{G, h}$ exists in $G$, no such tSCC exists in $G_{h}$ by Lemma A.4 and thus all vertices in $A_{G, h}$ and all elements in $Z$ are reachable from some vertex of $B_{G, h}$ in $G_{h}$ and hence contained in $F_{G, h}\left(r_{G, h}\right)$ (or for vertex connectivity and $\left|B_{G, h}\right|<k$ in $F_{G, h, w}\left(r_{G, h, w}\right)$ for all $w \in B_{G, h}$ ). As we exclude the case that $B_{G, h} \backslash Z$ is empty (for vertex connectivity), we have that in the case $\left|B_{G, h}\right|<k$ there exists some $w \in B_{G, h}$ such that $r_{G, h, w} \notin Z$. Thus by Lemma A.1 $Z$ is a $k$-dominator in $F_{G, h}\left(r_{G, h}\right)$, or for vertex connectivity and $\left|B_{G, h}\right|<k$, in $F_{G, h, w}\left(r_{G, h, w}\right)$ for $w \in B_{G, h} \backslash Z$.

\section{A.2 The algorithms for keSCCs and kvSCCs}

In this subsection we present our algorithms based on specific subgraphs $G_{h}$ that allow us to identify tSCCs and $k$-almost tSCCs in $G$ that have at most a certain size. This is crucial for the runtime analysis of the hierarchical graph decomposition technique. We first provide intuition for the runtime analysis and describe the algorithms and then formally prove their correctness and running times.

Let $G=(V, E)$ be a simple directed graph. We consider the following hierarchical graph decomposition: For level $i \in \mathbb{N}$ let the subgraph $G_{i}=\left(V, E_{i}\right)$ of $G$ contain all vertices in $V$ and for each vertex of $V$ its first $2^{i}$ incoming edges in $E$ (for some arbitrary but fixed ordering of the incoming edges of each vertex). Note that for $i \geq \log \left(\max _{v \in V} \operatorname{Indeg}_{G}(v)\right)$ we have $G_{i}=G$. Following the definitions in the previous subsection, let $A_{G, i}$ be the set of vertices with in-degree at most $2^{i}$ in $G$ and let $B_{G, i}=V \backslash A_{G, i}$ be the set of vertices with in-degree more than $2^{i}$ in $G$. 
Recall that we make progress in our algorithms by separating specific $k$-isolated sets, namely tSCCs and $k$-almost tSCCs, from the remaining graph. The main idea of the hierarchical graph decomposition is to detect "vertices to separate" that contain $O\left(2^{i}\right)$ vertices in $G_{i}$ in time proportional to the number of edges in $G_{i}$, i.e., in time $O\left(n \cdot 2^{i}\right)$. The search for "vertices to separate" is started at level $i=1$. When the search is not successful, the level $i$ is increased by one. Thus if the level has to be increased up to $i^{*}$ to identify a set of vertices, then this set contains $\Omega\left(2^{i^{*}}\right)$ vertices because otherwise it would have been detected already at level $i^{*}-1$. The time spent in the levels 1 up to $i^{*}$ forms a geometric series and thus can be bounded by $O\left(n \cdot 2^{i^{*}}\right)$ if the work per level is $O\left(n \cdot 2^{i}\right)$. In our algorithm we recurse on the identified $k$-isolated sets. To account for the recursion, we search "in parallel" on $G$ and its reverse graph $\operatorname{Rev}(G)$, that is, we also search for bSCCs and $k$-almost bSCCs by searching for tSCCs and $k$-almost tSCCs in $\operatorname{Rev}(G)$. The search for both ( $k$-almost) top and bottom SCCs ensures that whenever a ( $k$-almost) tSCC and a disjoint ( $k$-almost) bSCC exist in $G$, we only spend time proportional to the smaller one; that is, to identify a set $S$ when the per-level runtime is $O\left(n \cdot 2^{i}\right)$, we spend time $O(n \cdot \min \{|S|,|V \backslash S|\})$.

Let $T$ be a set of at most $2^{i}-k$ vertices such that there exists a $k$-almostTSCC $(T, Z, G)$ for some set of elements $Z$. Since the only edges from vertices of $V \backslash S$ to $S$ are, for edge connectivity, the edges in $Z$, or, for vertex connectivity, edges from $Z$, the in-degree of each vertex in $T$ can be at most $2^{i}$. Thus the vertices in $T$ are contained in $A_{G, i}$. This allows us to apply the results from the previous subsection to show that we can identify a tSCC or $k$-almost tSCC of $G$ with at most $2^{i}-k$ vertices by searching for tSCCs in $G_{i}$ and for $k$-dominators in derived flow graphs $F_{G, i}\left(r_{G, i}\right)$ as in Definitions A.7 and A.8.

Lemma A.12 (Extension of Lemma 4.1). Let $G$ be a simple directed graph.

(1) If a set of vertices $S$ with $|S| \leq 2^{i}+1$ induces a $t S C C G[S]$ in $G$,

(2) or if there is a set of elements $Z$ with $|Z|<k$ such that for some set of vertices $S$ with $|S| \leq 2^{i}-k+2$ there exists a $k$-almost $t S C C G[S]$ with respect to $Z$ in $G$, then $S \subseteq A_{G, i}$.

Proof. (1) Consider any graph $\tilde{G}$ in which $S$ induces a tSCC. Since a tSCC has no incoming edges, all incoming edges of vertices in $S$ have to come from other vertices of $S$. Thus each vertex in $S$ can have an in-degree of at most $|S|-1$ in $\tilde{G}$. The claim follows for $\tilde{G}=G$.

(2) Consider Case (1) for $\tilde{G}=G \backslash Z$. In $G$ each vertex in $S$ can have at most $k-1$ additional incoming edges compared to $G \backslash Z$, namely an edge from each vertex of $Z$ for vertex connectivity and the edges in $Z$ for edge connectivity. Thus we can bound the in-degree in $G$ of each vertex in $S$ by $2^{i}$. We have $S \subseteq A_{G, i}$.

Let $\gamma$ be the minimum of $\max _{v \in V} \operatorname{Indeg}_{G}(v)$ and $\max _{v \in V} \operatorname{Outdeg}_{G}(v)$. In Algorithm KSCC we start the search for ( $k$-almost) top SCCs at $i=1$. Whenever the search is not successful, we increase $i$ by one, until we have $2^{i} \geq \gamma$, that is, $G_{i}=G$ or $\operatorname{Rev}(G)_{i}=\operatorname{Rev}(G)$. For the search the Procedure kIsolatedSetLevel is used as long as $2^{i}<\gamma$, i.e., both $B_{G, i}$ and $B_{\operatorname{Rev}(G), i}$ are not empty, and the Procedure kIsolatedSet is used afterwards. If a tSCC of $G$ or $\operatorname{Rev}(G)$ is identified, the procedures return the set of vertices in the tSCC (and an empty set $Z)$. If a $k$-almost tSCC $k$-almostTSCC $(S, Z, G)$ or $k$-almostTSCC $(S, Z, \operatorname{Rev}(G))$ is identified, the procedures return the sets $S$ and $Z$. When one of the procedures returns a 
non-empty set $S$ and a (potentially empty) set $Z$, the algorithm recurses on each of $G[S]$ and $G[V \backslash S]$ for edge connectivity and on each of $G[S \cup Z]$ and $G[V \backslash S]$ for vertex connectivity. As the set $Z$ is not used further for edge connectivity, we do not have to worry about the direction of the edges in $Z$. We define the following shortcuts.

Definition A.13. For a set of vertices $S$ and a set of elements $Z$, let $G_{S, Z}$ for vertex connectivity be equal to $G[S \cup Z]$ and for edge connectivity equal to $G[S]$. Let $G_{V \backslash S}$ be equal to $G[V \backslash S]$.

If all the calls to the procedures return only empty sets, then the considered graph $G$ is a kSCC and is returned.

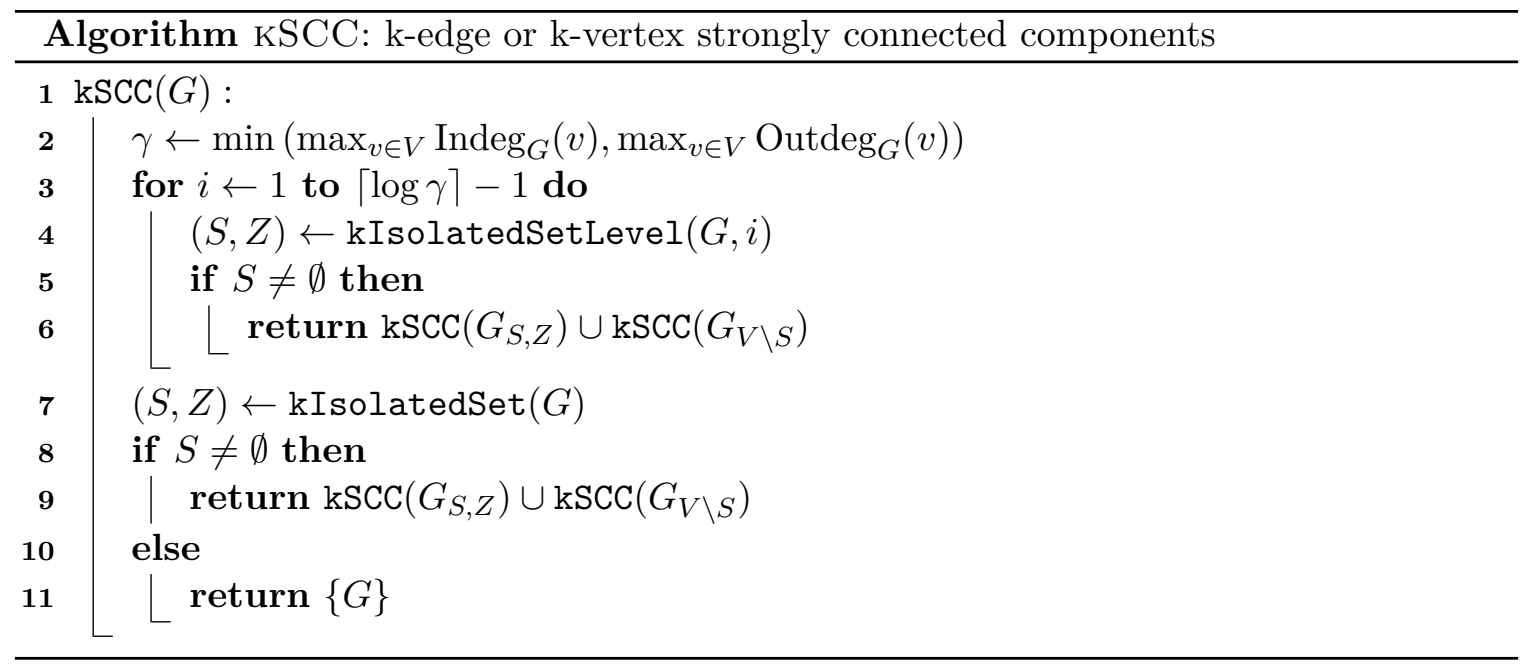

We use Procedure TopSCCWithout $(H, B)$ to denote the search for a tSCC induced by vertices $S$ in a graph $H$ such that $S$ does not contain a vertex of $B$. Such a tSCC can simply be found by marking tSCCs in a standard SCC algorithm. We let all procedures that search for an SCC return the set of vertices in the SCC.

The Procedure kIsolatedSetLevel first constructs the graph $\mathcal{G}_{i} \in\left\{G_{i}, \operatorname{Rev}(G)_{i}\right\}$ and searches for a tSCC in $\mathcal{G}_{i}$ that does not contain a vertex of $B_{\mathcal{G}, i}$, i.e., only contains vertices of $A_{G, i}$ for which we know that all their incoming edges in $\mathcal{G} \in\{G, \operatorname{Rev}(G)\}$ are present in $\mathcal{G}_{i}$. If such a tSCC is found, its set of vertices is returned (and an empty set is returned as the second return value). Otherwise the procedure continues in one of two ways. First consider edge connectivity and the case $\left|B_{\mathcal{G}, i}\right| \geq k$ for vertex connectivity. Then the flow graph $F_{\mathcal{G}, i}\left(r_{\mathcal{G}, i}\right)$ is constructed and searched for $k$-dominators. To find $k$-dominators the known algorithms listed in Section 5 are used. If a $k$-dominator $Z$ is found, a tSCC in $\mathcal{G}_{i} \backslash Z$ that does not contain a vertex of $B_{\mathcal{G}, i}$ is found and the vertices in the tSCC and the set $Z$ are returned. Otherwise the procedure returns two empty sets. In the case $\left|B_{\mathcal{G}, i}\right|<k$ for vertex connectivity for each $w \in B_{\mathcal{G}, i}$ the flow graph $F_{\mathcal{G}, i, w}\left(r_{\mathcal{G}, i, w}\right)$ is constructed and searched for a $k$-dominator. If at least one of the flow graphs contains a $k$-dominator $Z$, then a tSCC in $\mathcal{G}_{i} \backslash Z$ that does not contain a vertex of $B_{\mathcal{G}, i}$ is found and the vertices in the tSCC and the set $Z$ are returned. If none of the flow graphs contains a $k$-dominator, there could still exist a $k$-separator that contains all vertices of $B_{\mathcal{G}, i}$, which we also want to detect at this level. To this end, we first test whether $\mathcal{G}_{i} \backslash B_{\mathcal{G}, i}$ is strongly connected and return the vertices in 
a tSCC of $\mathcal{G}_{i} \backslash B_{\mathcal{G}, i}$ if not. If $\mathcal{G}_{i} \backslash B_{\mathcal{G}, i}$ is strongly connected and $\left|B_{\mathcal{G}, i}\right|<k-1$, we search for a $\left(k-\left|B_{\mathcal{G}, i}\right|\right)$-separator $Z^{\prime}$ in $\mathcal{G}_{i} \backslash B_{\mathcal{G}, i}$, using the known algorithm to find $k$-separators as described in Section 5. If such a $Z^{\prime}$ exists, then $Z=Z^{\prime} \cup B_{\mathcal{G}, i}$ is a $k$-separator and we return the vertices in a tSCC of $\mathcal{G}_{i} \backslash Z$ and the set $Z$.

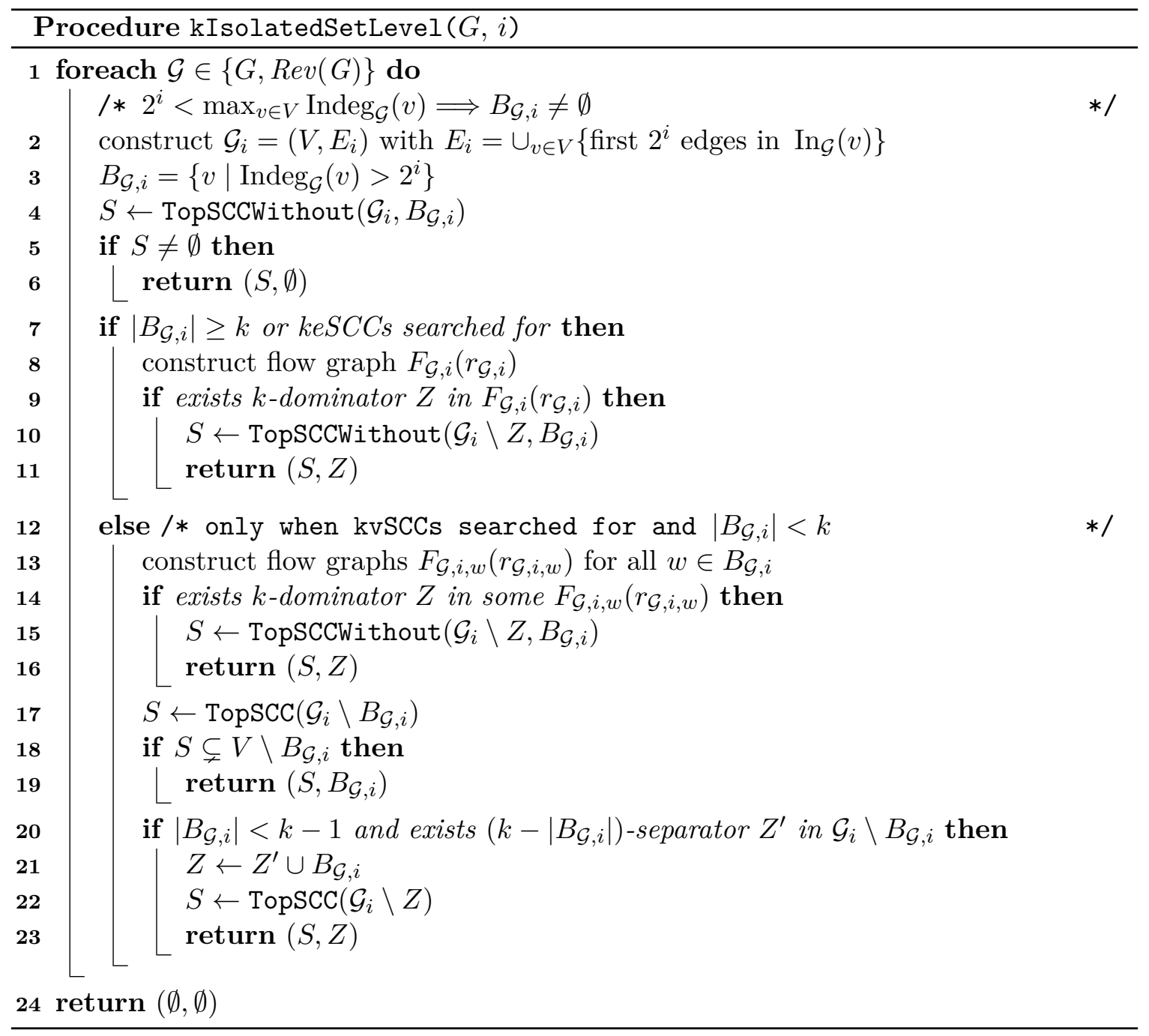

If Procedure kIsolatedSetLevel returns only empty sets for all levels $i<\log \gamma$, Procedure kIsolatedSet is called. It first tests whether the graph $G$ is strongly connected and returns a tSCC of $G$ if not. If $G$ is strongly connected, it searches for a $k$-separator in $G$. If a $k$-separator $Z$ is found, disjoint top and bottom SCCs exist in $G \backslash Z$. The procedure returns a tSCC of $G \backslash Z$ and the set $Z$ in this case. If the graph $G$ is strongly connected and does not contain a $k$-separator, then $G$ is a kSCC. In this case the procedure returns two empty sets, this branch of recursion stops in Algorithm $\mathrm{KSCC}$, and $\operatorname{kSCC}(G)$ returns $G$. 


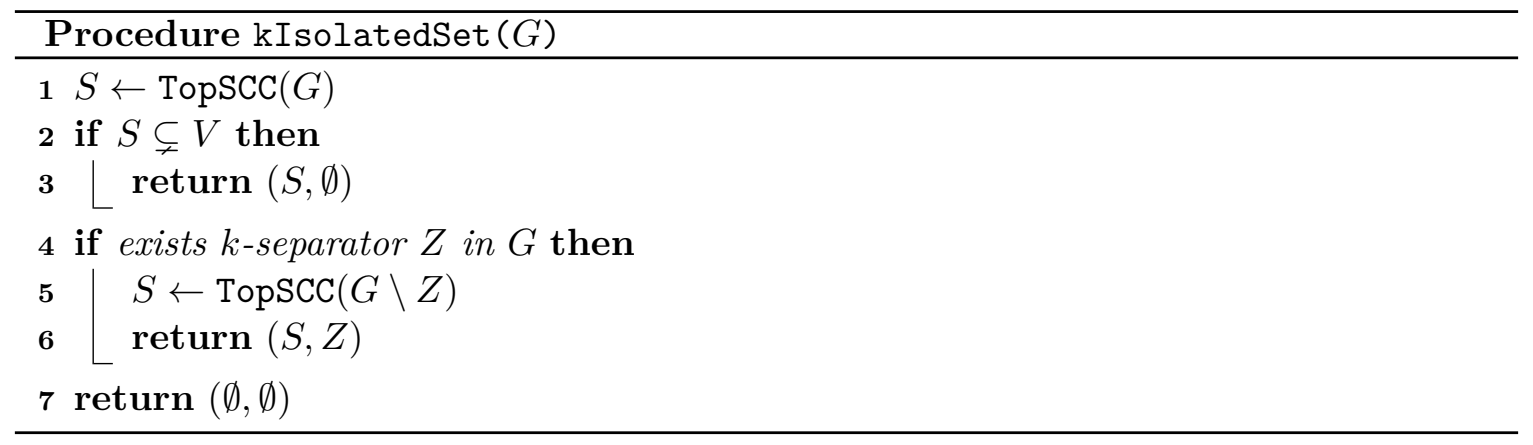

\section{A.2.1 Correctness}

Note that, for each of $\mathcal{G} \in\{G, \operatorname{Rev}(G)\}, \mathcal{G}_{i}$ is a subgraph of $\mathcal{G}$ such that all vertices of $\mathcal{G}_{i}$ that are missing incoming edges in $\mathcal{G}_{i}$ are contained in $B_{\mathcal{G}, i}$. Recall that the flow graph $F_{\mathcal{G}, i}\left(r_{\mathcal{G}, i}\right)$ is specified in Definitions A.7 and A.8 and further that for a non-empty set $S$ and a (potentially empty) set $Z$ returned by Procedure kIsolatedSetLevel or kIsolatedSet $G_{V \backslash S}$ is equal to $G[V \backslash S]$ and $G_{S, Z}$ is equal to $G[S]$ for edge connectivity and equal to $G[S \cup Z]$ for vertex connectivity.

To show the correctness of the algorithms for kSCCs, the following three parts are needed: (1) Every step in the algorithm can be executed as described. (2) Whenever a non-empty set $S$ and a set $Z$ are identified, every kSCC of $G$ is completely contained in either $G_{S, Z}$ or $G_{V \backslash S}$ and both $G_{S, Z}$ and $G_{V \backslash S}$ are not empty and thus proper subgraphs of $G$. (3) Whenever no non-empty set $S$ is identified in $G$, then $G$ is a kSCC.

We introduce Lemma A.14 and Theorem A.15 to show Parts (2) and (3), respectively. For Part (1) we need in particular that (1a) $B_{G, i}$ and $B_{R e v(G), i}$ are not empty whenever Procedure kIsolatedSetLevel $(G, i)$ is called and that (1b) whenever a $k$-dominator in a flow graph derived from $\mathcal{G}_{i}$ is identified, then there exists a tSCC in $\mathcal{G}_{i} \backslash Z$ that does not contain a vertex of $B_{\mathcal{G}, i}$. For (1a) the algorithm ensures $i<\log \gamma$, i.e., $2^{i}<\max _{v \in V} \operatorname{Indeg}_{G}(v)$ and $2^{i}<\max _{v \in V} \operatorname{Outdeg}_{G}(v)$. Lemma A.2 implies (1b).

The following lemma shows, based on the results of Subsection A.1, that the Procedures kIsolatedSetLevel and kIsolatedSet indeed find tSCCs or $k$-almost tSCCs in $G$ or $\operatorname{Rev}(G)$. This will imply by Lemma A.1 that every $\mathrm{kSCC}$ of $G$ is completely contained in either $G_{S, Z}$ or $G_{V \backslash S}$ whenever kSCC is called recursively on $G_{S, Z}$ and $G_{V \backslash S}$.

Lemma A.14. Let $0<i<\log \gamma$. If (1) Procedure kIsolatedSetLevel or (2) Procedure kIsolatedSet return a non-empty set $S$ and a set $Z$ (with $|Z|<k)$ for graph $G$, then $S$ induces either a top or a bottom SCC or a k-almost top or bottom SCC with respect to $Z$ in $G$. Additionally, the set $V \backslash(S \cup Z)$ is not empty.

Proof. (1) Let $\mathcal{G} \in\{G, \operatorname{Rev}(G)\}$ be the graph in which $S$ and $Z$ are identified. In Procedure kIsolatedSetLevel a set of vertices $S$ and a set of elements $Z$ can be identified in three ways: (a) $\mathcal{G}[S]$ is a tSCC in $\mathcal{G}_{i}$ that does not include vertices of $B_{\mathcal{G}, i}$ and $Z=\emptyset$; (b) the algorithm finds a $k$-dominator $Z$ in $F_{\mathcal{G}, i}\left(r_{\mathcal{G}, i}\right)$ or $F_{\mathcal{G}, i, w}\left(r_{\mathcal{G}, i, w}\right)$ for some $w \in B_{\mathcal{G}, i}$, and $\mathcal{G}[S]$ is a tSCC in $\mathcal{G}_{i} \backslash Z$ that does not include a vertex of $B_{\mathcal{G}, i} ;$ or (c) vertex connectivity is considered, we have $\left|B_{\mathcal{G}, i}\right|<k$, and either (c1) $\mathcal{G}_{i} \backslash B_{\mathcal{G}, i}$ is not strongly connected or (c2) there exists a $\left(k-\left|B_{\mathcal{G}, i}\right|\right)$-separator $Z^{\prime}$ in $\mathcal{G}_{i} \backslash B_{\mathcal{G}, i}$ for $k-\left|B_{\mathcal{G}, i}\right|>1$. Let $Z^{\prime}=\emptyset$ in 
Case (c1) and let $Z=Z^{\prime} \cup B_{\mathcal{G}, i}$. Then in Case (c) $S$ induces a tSCC in $\mathcal{G}_{i} \backslash Z$. We use that Case (b) can only occur if Case (a) did not occur and that Case (c) can only occur if the Cases (a) and (b) did not occur in this call to Procedure kIsolatedSetLevel. With this we have: In Case (a) $\mathcal{G}[S]$ is a tSCC in $\mathcal{G}$ by Lemma A.4. In Case (b) $k$-almostTSCC $(S, Z, \mathcal{G})$ exists by Corollary A.10. In Case (c) $k$-almostTSCC $(S, Z, \mathcal{G})$ exists by Lemma A.9.

In the Cases (a) and (b) we have $B_{\mathcal{G}, i} \cap V \backslash(S \cup Z) \neq \emptyset$ and thus both $S$ and $V \backslash(S \cup Z)$ are not empty. In Case (c1) $V \backslash(S \cup Z)=\emptyset$ is explicitly avoided by testing whether $S$ is a proper subset of $V \backslash Z$. In Case (c2) $V \backslash(S \cup Z) \neq \emptyset$ follows from the definition of $k$-separators.

(2) In Procedure kIsolatedSet a non-empty set of vertices $S$ and a set of elements $Z$ can be identified in two ways: (a) $G[S]$ is a top SCC in $G$ with $S \neq V$ and $Z=\emptyset$; or (b) $G$ is strongly connected, the algorithm finds a $k$-separator $Z$ in $G$ and $G[S]$ is a $k$-almost top SCC w.r.t. $Z$ in $G$. In both cases it clearly holds that $V \backslash(S \cup Z) \neq \emptyset$ because $G \backslash Z$ contains a top and a bottom SCC that are disjoint and only the tSCC is contained in $G[S]$.

Theorem A.15 (Correctness). Let $G$ be a simple directed graph. $\operatorname{kSCC}(G)$ computes the $k S C C s$ of $G$.

Proof. We denote by $G$ the graph for which $\operatorname{kSCC}(G)$ is called, which is equal to the input graph for the initial call to kSCC and equal to a subgraph of the input graph for all recursive calls to kSCC. Whenever $\operatorname{kSCC}(G)$ identifies a set $S$ and recursively calls itself on $G_{S, Z}$ and $G_{V \backslash S}$, then by Lemma A.14 the set $S$ either (a) induces a top or bottom SCC in $G$ or (b) a $k$-almost top or bottom SCC with respect to the set $Z$ in $G$. In Case (a) each kSCC is completely contained in either $G_{S, Z}$ or $G_{V \backslash S}$ by the fact that every kSCC is strongly connected. In Case (b) by Lemma A.1 no vertex of $V \backslash(S \cup Z)$ is $k$-connected to any vertex of $S$ in $\mathcal{G}$; thus each kSCC is completely contained in either $G_{S, Z}$ or $G_{V \backslash S}$ by the definition of a kSCC. This implies that each kSCC of $G$ is a $\mathrm{kSCC}$ in $G_{S, Z}$ or $G_{V \backslash S}$. Note that each kSCC of a subgraph of $G$ is by definition a subset of a kSCC of $G$. Hence the union of the kSCCs of $G_{S, Z}$ and $G_{V \backslash S}$ yields the kSCCs of $G$. Furthermore, by Lemma A.14 both $S$ and $V \backslash(S \cup Z)$ are not empty, i.e., the recursion is on proper subgraphs of $G$ and thus the algorithm terminates.

To show that the algorithm does not terminate until it has correctly identified all kSCCs of the input graph, it remains to show that whenever $\operatorname{ksCC}(G)$ is called, it either identifies a set $S$ and recursively calls itself on $G_{S, Z}$ and $G_{V \backslash S}$ or the graph $G$ is strongly connected and does not contain a $k$-separator, i.e., the graph $G$ is a kSCC. For this it is sufficient to show that if the algorithm has not identified a set $S$ in the for-loop, then it either identifies a set by the call to Procedure kIsolatedSet or $G$ is strongly connected and does not contain a $k$-separator. If $G$ is not strongly connected, then Procedure kIsolatedSet returns a tSCC in $G$. If $G$ is strongly connected, then either $G$ does not contain $k$-separators or Procedure kIsolatedSet finds a $k$-separator $Z$ and identifies a tSCC in $G \backslash Z$, which exists by the definition of a $k$-separator. 


\section{A.2.2 Runtime}

We already showed by Lemma A.12 that sets of at most $2^{i}-k+2$ vertices that induce a tSCC or a $k$-almost tSCC in $G$ are contained in $A_{G, i}=V \backslash B_{G, i}$, i.e., the incoming edges of each vertex in such a set are present in $G_{i}$. This allows us to apply the results from Subsection A.1 to show that our algorithms find such a set whenever one exists. Let $\gamma$ be the minimum of $\max _{v \in V} \operatorname{Indeg}_{G}(v)$ and $\max _{v \in V} \operatorname{Outdeg}_{G}(v)$ as in Algorithm KSCC.

Lemma A.16 (Extension of Lemma 4.2). If for some integer $1 \leq i<\log \gamma$ and $\mathcal{G} \in$ $\{G, \operatorname{Rev}(G)\}$ there exists a set of vertices $T \subseteq A_{\mathcal{G}, i}$ that either induces a $t S C C$ or a $k$-almost tSCC with respect to some set of elements $Z$ with $|Z|<k$ and $T \subsetneq V \backslash Z$ in $\mathcal{G}$, then kIsolatedSetLevel $(G, i)$ returns a non-empty set $S$.

Proof. If $\mathcal{G}[T]$ is a tSCC in $\mathcal{G}$, then $\mathcal{G}_{i}[T]$ is a tSCC without vertices of $B_{\mathcal{G}, i}$ in $\mathcal{G}_{i}$ by Lemma A.4. Thus in this case in Line 4 of Procedure kIsolatedSetLevel a non-empty set $S$ is returned. In the following assume that no non-empty set $S^{\prime}$ is identified in Line 4, i.e., that no tSCC without vertices of $B_{\mathcal{G}, i}$ exists in $\mathcal{G}_{i}$.

If $\mathcal{G}[T]$ is a $k$-almost tSCC with respect to a set of elements $Z$ in $\mathcal{G}$, then either $B_{\mathcal{G}, i} \backslash Z$ is empty or a non-empty set $S$ is by Corollary A.11 returned in Line 10 or, for vertex connectivity and $\left|B_{\mathcal{G}, i}\right|<k$, in Line 15 .

If $B_{\mathcal{G}, i} \backslash Z$ is empty, i.e., $Z \supseteq B_{\mathcal{G}, i}$, assume that no $k$-dominator is found in any flow graph, i.e., no non-empty set $S^{\prime}$ is returned before Line 17 . If $Z=B_{\mathcal{G}, i}$, then $\mathcal{G}_{i}[T]$ is a tSCC in $\mathcal{G}_{i} \backslash B_{\mathcal{G}, i}$ and a non-empty set $S$ is identified in Line 17. Otherwise $Z^{\prime}=Z \backslash B_{\mathcal{G}, i} \neq \emptyset$ contains less than $k-\left|B_{\mathcal{G}, i}\right|$ vertices and $k$-almostTSCC $\left(T, Z^{\prime}, \mathcal{G}_{i} \backslash B_{\mathcal{G}, i}\right)$ exists. If no non-empty set was identified in Line 17 , then $\mathcal{G}_{i} \backslash B_{\mathcal{G}, i}$ is strongly connected. Thus, since $T \subsetneq V \backslash Z$, the set $Z^{\prime}$ is a $\left(k-\left|B_{\mathcal{G}, i}\right|\right)$-separator in $\mathcal{G}_{i} \backslash B_{\mathcal{G}, i}$. Hence a non-empty set $S$ is returned in Line 22.

Corollary A.17. Let $(a) i^{*}<\log \gamma$ be the smallest integer such that for all $1 \leq i<i^{*}$ kIsolatedSetLevel $(G, i)$ returned empty sets $S^{\prime}$ and a non-empty set $S$ is returned by $\mathrm{kIsolatedSetLevel}\left(G, i^{*}\right)$ or, (2) if all calls to $\mathrm{kIsolatedSetLevel}(G, i)$ returned empty sets $S^{\prime}$ for $1 \leq i<\log \gamma$, but $\mathrm{kIsolatedSet}(G)$ returned a non-empty set $S$, let $i^{*}=\lceil\log \gamma\rceil$. Then $|S|>2^{i^{*}-1}-k+2$, respectively.

Proof. By Lemma A.14 the set $S$ either induces a tSCC or a $k$-almost tSCC with $S \subsetneq$ $V \backslash Z$ in $\mathcal{G}$, where $\mathcal{G}$ is either $G$ or $\operatorname{Rev}(G)$ and $Z$ is the second argument returned by kIsolatedSetLevel $\left(G, i^{*}\right)$ or kIsolatedSet $(G)$. By Lemma A.16 we have $S \cap B_{\mathcal{G}, i^{*}-1} \neq \emptyset$. The claim then follows from Lemma A.12.

In the runtime analysis we argue that whenever the search for a set $S$ stops at a certain level $i^{*}$, the algorithm has spent time at most proportional to the number of vertices in the smaller set of $S$ and $V \backslash S$. Additional to Corollary A.17, we use the fact that if a graph is not strongly connected, it contains a top and a bottom SCC that are disjoint, which implies that one of them contains at most half of the vertices of the graph.

Theorem A.18 (Runtime keSCCs). Algorithm $\mathrm{kSCC}$ for keSCCs can be implemented in time $O\left(n^{2} \log n\right)$ for any integral constant $k>2$ and in time $O\left(n^{2}\right)$ for $k=2$.

Proof. We denote by $n$ and $m$ the number of vertices and edges in the input graph and by $n^{\prime}$ the number of vertices in the graph $G$ of the current level of the recursion in Algorithm KSCC. 
Let $\gamma$ denote the minimum of $\max _{v \in V} \operatorname{Indeg}_{G}(v)$ and $\max _{v \in V} \operatorname{Outdeg}_{G}(v)$. Let $S$ be a nonempty set of vertices returned by kIsolatedSetLevel or kIsolatedSet.

To efficiently construct the graphs $\mathcal{G}_{i}$ for $1 \leq i<\lceil\log \gamma\rceil$ and $\mathcal{G} \in\{G, \operatorname{Rev}(G)\}$ we maintain for all vertices $w$ a list of $\operatorname{In}(w)$ and a list of $\operatorname{Out}(w)$. We do not update this data structure immediately when $G$ is split into $G[S]$ and $G[V \backslash S]$ but remove obsolete entries in $\operatorname{In}(w)$ and $\operatorname{Out}(w)$ whenever we encounter them while constructing $\mathcal{G}_{i}$. This can happen at most once for each entry and thus takes total time $O(m) \in O\left(n^{2}\right)$.

We first analyze the time per iteration of the for-loop in $\operatorname{kSCC}(G)$. In iteration $i$ the algorithm calls kIsolatedSetLevel $(G, i)$. Searching on both $G$ and $\operatorname{Rev}(G)$ only increases the running time by a factor of two; we analyze the search on $G$, the search on $\operatorname{Rev}(G)$ is analogous. Given the above data structure, constructing $G_{i}, F_{G, i}$, and determining $B_{G, i}$ takes time $O\left(2^{i} \cdot n^{\prime}\right)$. Finding SCCs in $G_{i}$ takes time linear in the number of edges in $G_{i}$, i.e., time $O\left(2^{i} \cdot n^{\prime}\right)$. Finding $k$-dominators in $F_{G, i}\left(r_{G, i}\right)$ takes time $O\left(2^{i} \cdot n^{\prime} \cdot \log n^{\prime}\right)$ for $k>2$ and time $O\left(2^{i} \cdot n^{\prime}\right)$ for $k=2$. We give below the analysis for $k>2$, the analysis for $k=2$ is identical but without the log-factor. We have that kIsolatedSetLevel $(G, i)$ and hence iteration $i$ of the outer for-loop take time $O\left(2^{i} \cdot n^{\prime} \cdot \log n^{\prime}\right)$.

The search for SCCs and $k$-separators in kIsolatedSet can also be done in time proportional to the number of edges times $\log n$, which can be bounded by $O\left(\gamma \cdot n^{\prime} \cdot \log n^{\prime}\right)$.

Let $i^{*}<\lceil\log \gamma\rceil$ be the last iteration of $\operatorname{kSCC}(G)$, i.e., the iteration before $\operatorname{kSCC}(G)$ returns $G$ (Case (1)) or recursively calls itself on the subgraphs $G[S]$ and $G[V \backslash S]$ (Case (2)). The time spent in the iterations $i=1$ up to $i^{*}$ forms a geometric series that can be bounded by $O\left(2^{i^{*}} \cdot n^{\prime} \cdot \log n^{\prime}\right)$. If $i^{*}=\lceil\log \gamma\rceil-1$, we have $\gamma \leq 2^{i^{*}+1}$ and thus also the time spent in kIsolatedSet can be bounded with $O\left(2^{i^{*}} \cdot n^{\prime} \cdot \log n^{\prime}\right)$.

Case (1): If $\operatorname{kSCC}(G)$ returns $G$, the recursion stops. In this case the running time is proportional to $\gamma \cdot n^{\prime} \cdot \log n^{\prime} \leq\left(n^{\prime}\right)^{2} \cdot \log n^{\prime}$.

Case (2): In iteration $i^{*}-1$ no top or bottom SCC or $k$-almost top or bottom SCC was detected in $G_{i^{*}-1}$. Thus we have by Corollary A.17 that $|S|>2^{i^{*}-1}-k+2$. Let $\mathcal{G} \in\{G, \operatorname{Rev}(G)\}$ be the graph in which $S$ was detected at level $i^{*}$. If (a) $G$ is strongly connected, then $\mathcal{G}[S]$ is a $k$-almost tSCC in $\mathcal{G}$ with respect to some set of elements $Z$ and we have that there exists a $k$-almost bSCC in $\mathcal{G}$ with respect to $Z$. This $k$-almost bSCC is contained in $V \backslash S$ and by Lemmata A.12 and A.16 contains more than $2^{i^{*}-1}-k+2$ vertices, i.e., we have $|V \backslash S|>2^{i^{*}-1}-k+2$. If (b) $G$ is not strongly connected, then there exist a top and a bottom SCC in $G$ which are disjoint and both have more than $2^{i^{*}-1}-k+2$ vertices by Lemmata A.12 and A.16. Since $G[S]$ is a strongly connected subgraph of $G$, it has to be completely contained in either the top or the bottom SCC. Thus also in this case we have $|V \backslash S|>2^{i^{*}-1}-k+2$. Let $\min (|S|,|V \backslash S|)$ be denoted by $n_{e}$. By the definition of $n_{e}$ we have $n_{e} \leq n^{\prime} / 2$. By the analysis above, without the recursive calls, $\operatorname{kSCC}(G)$ spends time proportional to $n_{e} \cdot n^{\prime} \cdot \log n^{\prime}$.

We show next that the total time spent in all recursive calls to $\operatorname{kSCC}(G)$, and thus the total running time of Algorithm KSCC for keSCCs, is of order $f(n)=2 n^{2} \log n$. In Case (1), i.e., if the recursion stops, we have $f\left(n^{\prime}\right)=\left(n^{\prime}\right)^{2} \log n<2\left(n^{\prime}\right)^{2} \log n$. In Case (2) we have by 
induction, and in particular for $n^{\prime}=n$,

$$
\begin{aligned}
f\left(n^{\prime}\right) & \leq f\left(n_{e}\right)+f\left(n^{\prime}-n_{e}\right)+n_{e} n^{\prime} \log n^{\prime}, \\
& =2 n_{e}^{2} \log n^{\prime}+2\left(n^{\prime}-n_{e}\right)^{2} \log n^{\prime}+n_{e} n^{\prime} \log n^{\prime}, \\
& =2 n_{e}^{2} \log n^{\prime}+2\left(n^{\prime}\right)^{2} \log n^{\prime}-4 n_{e} n^{\prime} \log n^{\prime}+2 n_{e}^{2} \log n^{\prime}+n_{e} n^{\prime} \log n^{\prime}, \\
& =2\left(n^{\prime}\right)^{2} \log n^{\prime}+4 n_{e}^{2} \log n^{\prime}-3 n_{e} n^{\prime} \log n^{\prime}, \\
& \leq 2\left(n^{\prime}\right)^{2} \log n^{\prime},
\end{aligned}
$$

where the last inequality follows from $n_{e} \leq n^{\prime} / 2$.

Theorem A.19 (Runtime kvSCCs). Algorithm KSCC for kvSCCs can be implemented in time $O\left(n^{3}\right)$ for any integral constant $k>2$ and in time $O\left(n^{2}\right)$ for $k=2$.

Proof. The proof is analogous to the proof of Theorem A.18 except that (1) the recursion is on $G[S \cup Z]$ and $G[V \backslash S]$ whenever a $k$-almost top or bottom SCC with respect to $Z$ was found and (2) the time to find $k$-dominators and $k$-separators is proportional to the number of edges times the number of vertices in the graph for $k>2$.

To efficiently update the adjacency lists, we update the lists of the vertices in $Z$ for each of $G[S \cup Z]$ and $G[V \backslash S]$ immediately when the recursive call occurs, while for all other vertices the same argument as before applies.

As before, let $n^{\prime}$ denote the number of vertices in the graph $G$ in the current level of recursion in Algorithm $\mathrm{KSCC}$. Let $n_{v}=\min (|S|,|V \backslash S|-k+1)$. We first analyze the runtime for an arbitrary integral constant $k>2$ and then for $k=2$. Let $O\left(n_{v} \cdot\left(n^{\prime}\right)^{2}\right)$ be the runtime bound of $\operatorname{kSCC}(G)$ without the recursive calls whenever the recursion does not stop. Note that $n_{v} \leq n^{\prime} / 2$. We stop the recursion when the number of vertices $n^{\prime}$ is less than $14 k^{3}$, i.e., a constant; this can happen at most $n$ times. In this case we can use the known $O\left(m n^{2}\right)$-time algorithm for kvSCCs to compute the kvSCCs of $G$ in constant time. We obtain an upper bound of order $f(n)=n^{3}$ on the total runtime of the algorithm for kvSCCs as follows:

$$
\begin{aligned}
f\left(n^{\prime}\right) & \leq f\left(n_{v}+k\right)+f\left(n^{\prime}-n_{v}\right)+n_{v}\left(n^{\prime}\right)^{2}, \\
& =\left(n_{v}+k\right)^{3}+\left(n^{\prime}-n_{v}\right)^{3}+n_{v}\left(n^{\prime}\right)^{2}, \\
& =\left(n^{\prime}\right)^{3}-2 n_{v}\left(n^{\prime}\right)^{2}+3 n_{v}^{2} n^{\prime}+3 n_{v}^{2} k+3 n_{v} k^{2}+k^{3}, \\
& \leq\left(n^{\prime}\right)^{3}-2 n_{v}\left(n^{\prime}\right)^{2}+3 n_{v}^{2} n^{\prime}+7 k^{3} n_{v}^{2}, \\
& \leq\left(n^{\prime}\right)^{3} .
\end{aligned}
$$

For $k=2$ let $O\left(n_{v} \cdot n^{\prime}\right)$ be the runtime bound of $\operatorname{kSCC}(G)$ without the recursive calls whenever the recursion does not stop. We stop the recursion whenever the number of remaining vertices is at most 8 , i.e., a constant. In this case we use the $O(m n)$-algorithm to determine the 2 vSCCs of $G$ in constant time. With $3 n^{\prime} / 4 \geq 6 n_{v} / 5$ and $n^{\prime} / 4 \geq 2>9 / 5$ we have $5 n^{\prime} \geq 6 n_{v}+9$, which we use in the following to obtain an upper bound of order 
$f(n)=3 n^{2}$ on the total running time of Algorithm kSCC for kvSCCs. We have

$$
\begin{aligned}
f\left(n^{\prime}\right) & \leq f\left(n_{v}+1\right)+f\left(n^{\prime}-n_{v}\right)+n_{v} n^{\prime}, \\
& =3\left(n_{v}+1\right)^{2}+3\left(n^{\prime}-n_{v}\right)^{2}+n_{v} n^{\prime}, \\
& =3 n_{v}^{2}+6 n_{v}+3+3\left(n^{\prime}\right)^{2}-6 n_{v} n^{\prime}+3 n_{v}^{2}+n_{v} n^{\prime}, \\
& =3\left(n^{\prime}\right)^{2}+6 n_{v}^{2}-5 n_{v} n^{\prime}+6 n_{v}+3, \\
& \leq 3\left(n^{\prime}\right)^{2} .
\end{aligned}
$$

\section{B An $O\left(m^{2} / \log n\right)$-time algorithm for $2 \mathrm{eSCCs}$}

In this section we combine our results for almost tSCCs and dominators in subgraphs in Appendix A.1 with a local-search technique used for Büchi games by Chatterjee et al. [CJH03]. Throughout the section we only consider edge-connectivity and $k=2$. We use the definitions in Sections 2 and 5 but use the more common terms bridges and edge-dominators instead of 2 -separators and 2-dominators. In the algorithm we assume that each vertex in the input graph has constant in- and out-degree. We show how every graph with $m$ edges can be transformed in $O(m)$ time into a graph with in- and out-degree at most three with equivalent 2eSCCs, $\Theta(m)$ vertices, and $\Theta(m)$ edges. We are not aware of such a transformation for $2 \mathrm{vSCCs}$.

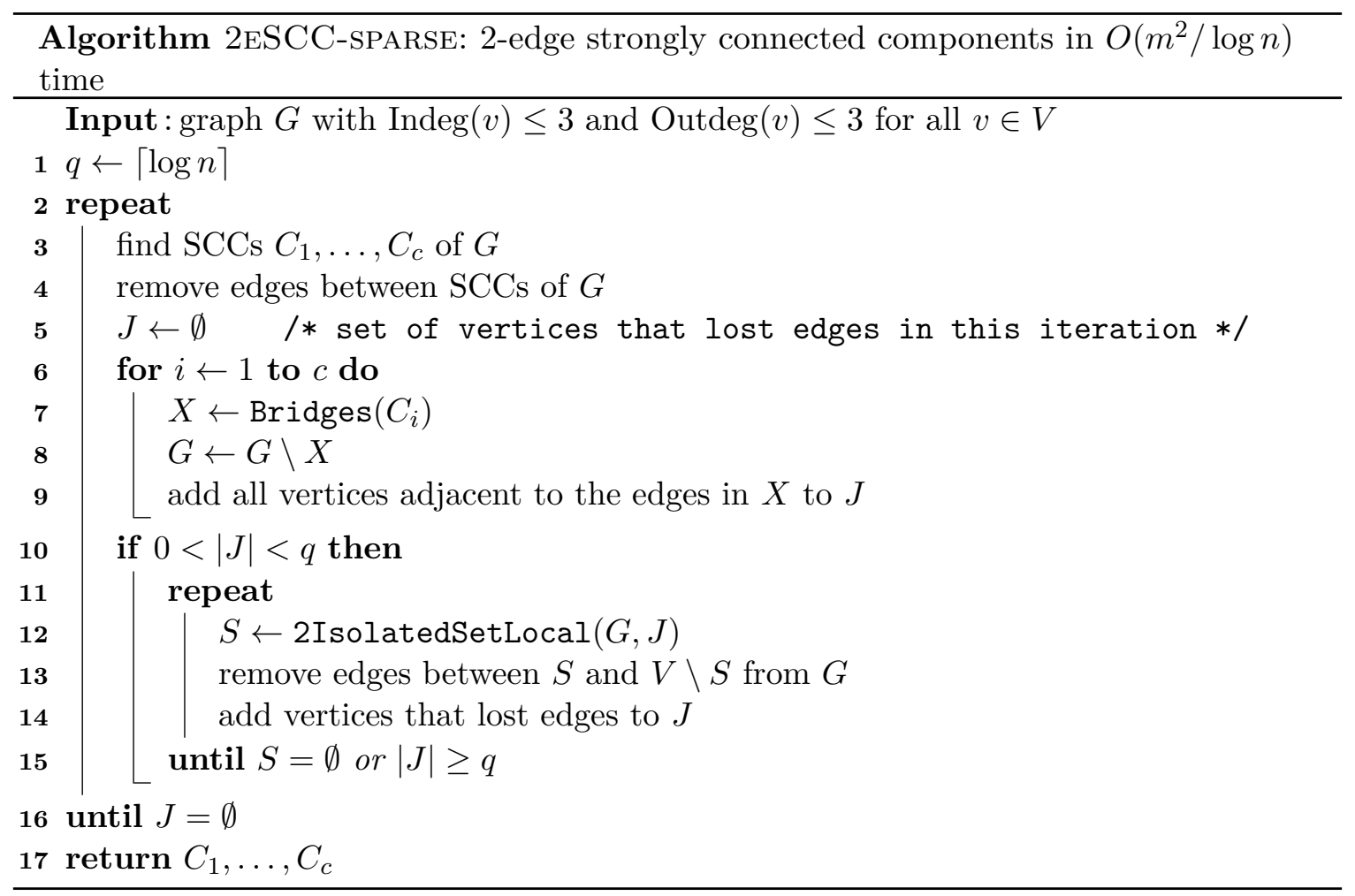

We first describe the algorithm and then prove its correctness and running time. In Algorithm 2ESCC-SPARSE the main repeat-until loop without the local searches is equivalent 
to the simple $O(m n)$-algorithm for 2eSCCs: First the strongly connected components are determined, then in each strongly connected component all bridges are identified and removed. Both operations take time $O(m)$. This is repeated until no more bridges are found. Our algorithm removes the bridges and the edges between the strongly connected components from the graph and stores all vertices that were adjacent to the removed edges in the set $J$. The set $J$ is empty at the beginning of each iteration of the main repeat-until loop. If no bridges are found in an iteration of the main repeat-until loop, the algorithm terminates. In this case each strongly connected component of the maintained graph $G$ is 2-edge strongly connected. If $J$ contains less than $q$ vertices, where $q$ is of order $\log n$, then Procedure 2IsolatedSetLocal is called. 2IsolatedSetLocal $(G, J)$ identifies a non-empty set of vertices that induces a top or bottom SCC or an almost top or bottom SCC in $G$ whenever there is one that contains a vertex in $J$ and has less than $d$ vertices, where $d$ is also of order $\log n$. If the procedure returns a non-empty set $S$, then the edges between $S$ and the remaining vertices $V \backslash S$ are removed from $G$ and the vertices that lost edges are added to $J$. The call to 2IsolatedSetLocal $(G, J)$ is repeated until either $J$ contains more than $q$ vertices or 2IsolatedSetLocal $(G, J)$ returns an empty set. Then a new iteration of the main repeat-until loop is started. Since vertices are added to $J$ only when they lost edges and $J$ is reset in each iteration of the main repeat-until loop, the set $J$ can contain $\Omega(\log n)$ vertices only $O(m / \log n)$ times. If 2IsolatedSetLocal $(G, J)$ returns an empty set, we can show that each top or bottom SCC or almost top or bottom SCC in the current graph $G$ contains $\Omega(\log n)$ vertices. In this case in the following iteration of the main repeat-until loop either at least two sets of vertices with $\Omega(\log n)$ vertices each are separated from each other (by deleting the edges between them) or the algorithm terminates. Thus also this case can happen at most $O(\mathrm{~m} / \log n)$ times. Hence the total time without the calls to Procedure 2IsolatedSetLocal can be bounded with $O\left(m^{2} / \log n\right)$. The constant-degree assumption allows us to bound the work done in Procedure 2IsolatedSetLocal.

The local searches in Procedure 2IsolatedSetLocal use the results presented in Appendix A.1 on subgraphs $G_{j}$ defined as follows. Let $j$ be a vertex of $J$ and let $d$ be a parameter set to $\lceil\varepsilon \log n\rceil$ for some $\varepsilon \in(0,1)$. Let $V_{G, j}$ be the set of vertices that have a path of length at most $d$ to $j$ in $G$. The subgraph $G_{j}$ is equal to $G\left[V_{G, j}\right]$. Following the definitions in Appendix A.1, the set of vertices $B_{G, j}$ is defined as all vertices in $V_{G, j}$ that have incoming edges from $V \backslash G_{j}$, i.e., for each vertex in $A_{G, j}=V_{G, j} \backslash B_{G, j}$ we know that all its incoming edges in $G$ are contained in the subgraph $G_{j}$. As in Definition A.7, let $F_{G, j}$ be the graph $G_{j}$ with all vertices in $B_{G, j}$ contracted to a new vertex $r_{G, j}$. We use the flow graph $F_{G, j}\left(r_{G, j}\right)$ to find edge-dominators and almost tSCCs with respect to them.

In Procedure 2IsolatedSetLocal $(G, J)$ we search for a tSCC or an almost tSCC in $\mathcal{G}_{j}$ for each $j \in J$ and each of $\mathcal{G} \in\{G, \operatorname{Rev}(G)\}$. As Algorithm 2ESCC-SPARSE makes progress by removing edges from the maintained graph $G$, we only want to identify (almost) tSCCs for which $G$ contains edges between the (almost) tSCC and the remaining graph. Note that it can easily happen that there exists a tSCC in $G$ without outgoing edges (i.e. a tSCC that is also a bSCC) because no vertex is removed from $J$ when Procedure 2IsolatedSetLocal $(G, J)$ is called repeatedly in the inner repeat-until loop of Algorithm 2ESCC-SPARSE. When Procedure 2IsolatedSetLocal $(G, J)$ returns a non-empty set of vertices $S$ (that induces a (almost) top or bottom SCC in $G$ ), the edges between the vertices in $S$ and the vertices in $V \backslash S$ are removed from $G$. We now describe the steps in Procedure 2IsolatedSetLocal $(G, J)$. First the vertices in $V_{\mathcal{G}, j}$ are identified by running a breadth-first search of depth $d$ from 


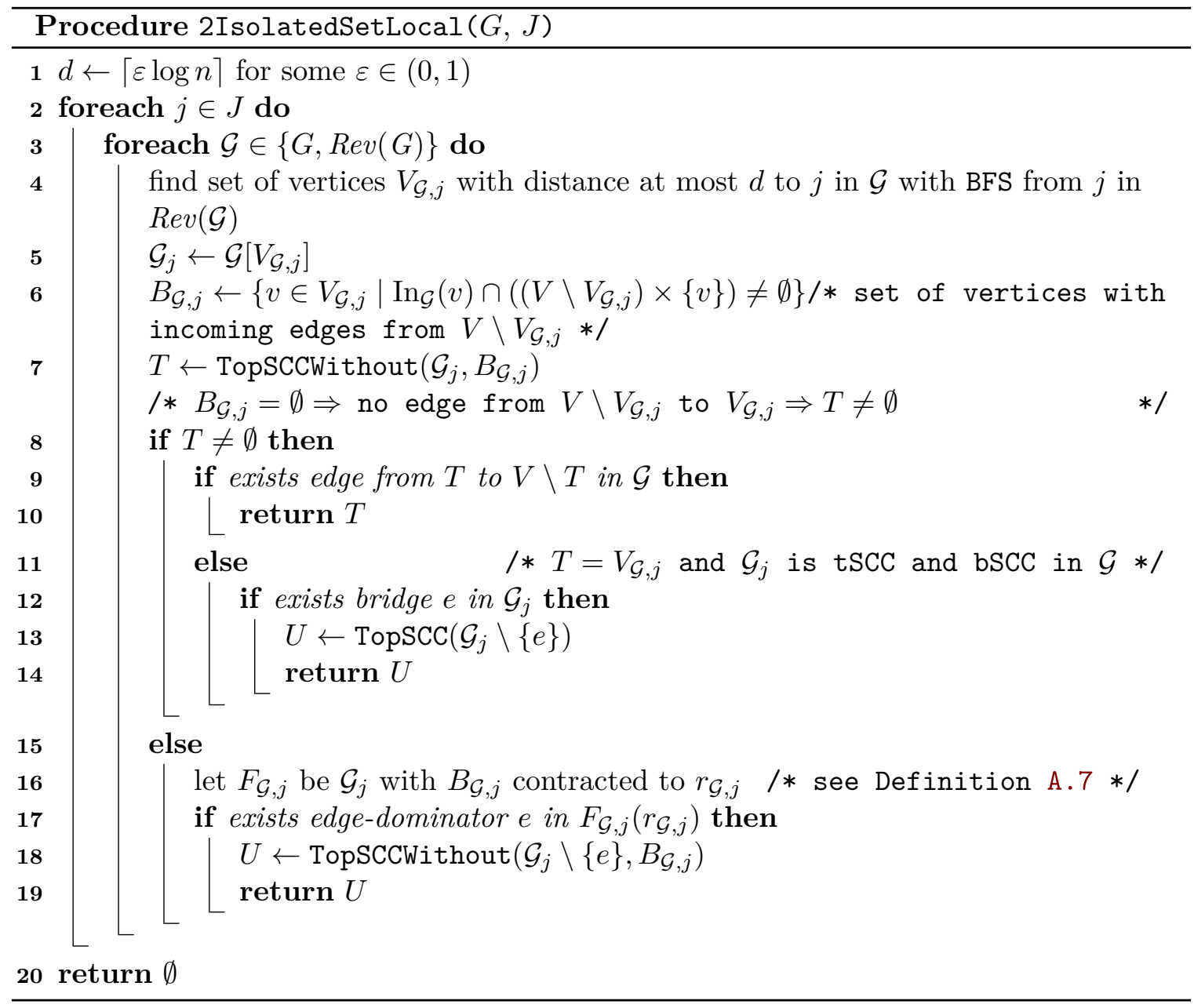


$j$ on $\operatorname{Rev}(\mathcal{G})$. Then a tSCC induced by a set of vertices $T$ contained in $A_{\mathcal{G}, j}=V_{\mathcal{G}, j} \backslash B_{\mathcal{G}, j}$ is searched in $\mathcal{G}_{j}$. If $T$ exists and $\mathcal{G}$ contains edges from $T$ to $V \backslash T$, then $T$ is returned. If $T$ exists but there are no edges from $T$ to $V \backslash T$ in $\mathcal{G}$, then $T$ also induces a bSCC in $\mathcal{G}$. Since all vertices in $V_{\mathcal{G}, j}$ are reachable from $j$, we have $T=V_{\mathcal{G}, j}$ and $B_{\mathcal{G}, j}=\emptyset$ in this case. Thus we (a) cannot make progress by separating $T$ from $V \backslash T$ and (b) cannot use the flow graph $F_{\mathcal{G}, j}\left(r_{\mathcal{G}, j}\right)$ for identifying almost tSCCs. However, since for each vertex in $V_{\mathcal{G}, j}$ all its incoming edges are contained in $\mathcal{G}_{j}$ and $\mathcal{G}_{j}$ is strongly connected, an almost tSCC in $\mathcal{G}_{j}$ can be found whenever one exists by searching for a bridge $e$ in $\mathcal{G}_{j}$ and a tSCC in $\mathcal{G}_{j} \backslash\{e\}$. If a bridge $e$ exists, the procedure returns the vertices in the tSCC. If no bridge exists, then $G_{j}$ is a $2 \mathrm{eSCC}$ and we do not have to consider the vertices in $V_{G, j}$ further. If no tSCC induced by vertices in $A_{\mathcal{G}, j}$ exists in $\mathcal{G}_{j}$, then each vertex in $\mathcal{G}_{j}$ can be reached from vertices of $B_{\mathcal{G}, j}$ and $B_{\mathcal{G}, j}$ is not empty. In this case we search for an edge-dominator in $F_{\mathcal{G}, j}\left(r_{\mathcal{G}, j}\right)$. If an edge-dominator $e$ exists, the procedure returns the vertices in a tSCC in $\mathcal{G}_{j} \backslash\{e\}$ that is induced by vertices of $A_{\mathcal{G}, j}$.

We show next how to transform a graph to a constant degree graph without changing the 2eSCCs. The transformation basically replaces each vertex $v$ such that $t_{v}=$ $\max (\operatorname{Indeg}(v), \operatorname{Outdeg}(v))>3$ by two cycles of $t_{v}$ many vertices, one cycle in each direction.

Lemma B.1. Let $G=(V, E)$ be a simple directed graph with an arbitrary fixed ordering in $\operatorname{In}(v)$ and $\operatorname{Out}(v)$ for each $v \in V$. Let $t_{v}=\max (\operatorname{Indeg}(v), \operatorname{Outdeg}(v))$. Let $\widetilde{G}$ be $G$ with every vertex $v \in V$ with $t_{v}>3$ replaced by $t_{v}$ vertices $V_{v}$ as follows. Let $v_{0}, \ldots, v_{t_{v}-1}$ be the vertices of $V_{v}$. We add for each $v_{i}$ an edge $\left(v_{i}, v_{(i+1) \bmod t_{v}}\right)$ and an edge $\left(v_{i}, v_{(i-1) \bmod t_{v}}\right)$ to $\widetilde{G}$. Additionally we add for each edge $(u, v) \in E$ that is the $i$-th edge in $\operatorname{Out}(u)$ and the $j$-th edge in $\operatorname{In}(v)$ an edge $\left(u_{i-1}, v_{j-1}\right)$ to $\widetilde{G}$. For vertices $v \in V$ with $t_{v} \leq 3$, let $V_{v}=\{v\}$. Then $G[S]$ is a $2 e S C C$ in $G$ if and only if $\widetilde{G}\left[\cup_{u \in S} V_{u}\right]$ is a $2 e S C C$ in $\widetilde{G}$.

Proof. Since every subgraph $\widetilde{G}\left[V_{v}\right]$ is 2-edge strongly connected, it is sufficient to show that there are two edge-disjoint paths from a vertex $u$ to a vertex $v \neq u$ in $G$ if and only if there are two edge-disjoint paths from some vertex $\widetilde{u} \in V_{u}$ to some vertex $\widetilde{v} \in V_{v}$ with $v \neq u$ in $\widetilde{G}$. We can assume w.l.o.g. that the edge-disjoint paths are simple.

$\Leftarrow$ : Let $\widetilde{P}_{1}$ and $\widetilde{P}_{2}$ be two edge-disjoint paths in $\widetilde{G}$ from a vertex $\widetilde{u} \in V_{u}$ to a vertex $\widetilde{v} \in V_{v}$ with $v \neq u$. We can construct two edge-disjoint paths $P_{1}$ and $P_{2}$ from $u$ to $v$ in $G$ by simply removing all edges that are contained in a subgraph $\widetilde{G}\left[V_{w}\right]$ for some $w \in V$ and replacing all vertices $\widetilde{w} \in V_{w}$ with $w$ in the remaining edges. As there is a one-to-one relation between edges between subgraphs $\widetilde{G}\left[V_{w}\right]$ and edges in $E$, the paths $P_{1}$ and $P_{2}$ must be edge-disjoint.

$\Rightarrow$ : Let $P_{1}$ and $P_{2}$ be two edge-disjoint paths from a vertex $u$ to a vertex $v \neq u$ in $G$. We construct two edge-disjoint paths $\widetilde{P}_{1}$ and $\widetilde{P}_{2}$ from a vertex $\widetilde{u} \in V_{u}$ to a vertex $\widetilde{v} \in V_{v}$ in $\widetilde{G}$. First add for all edges in the path $P_{\ell}, \ell \in\{1,2\}$, the corresponding edges between two different subgraphs $\widetilde{G}\left[V_{w}\right]$ in $\widetilde{G}$ to $\widetilde{P}_{\ell}$. It remains to connect the edges in $\widetilde{P}_{\ell}$ within subgraphs $\widetilde{G}\left[V_{w}\right]$ with $t_{w}>3$. For any $w$ that is only contained in one of $P_{1}$ and $P_{2}$, we can select some arbitrary path within $\widetilde{G}\left[V_{w}\right]$ that connects the edges in $\widetilde{P}_{\ell}$. Let $w$ be a vertex in both $P_{1}$ and $P_{2}$. Let $w_{0}, \ldots, w_{t_{w}-1}$ be the vertices of $V_{w}$. We connect the path $\widetilde{P}_{1}$ in the subgraph $\widetilde{G}\left[V_{w}\right]$ by using the edges in one direction, i.e., from $w_{i}$ to $w_{(i+1) \bmod t_{w}}$ for $0 \leq i<t_{w}$, and we connect the path $\widetilde{P}_{2}$ in the subgraph $\widetilde{G}\left[V_{w}\right]$ by using the edges in the other direction, i.e., from $w_{i}$ to $w_{(i-1) \bmod t_{w}}$. In this way the paths $\widetilde{P}_{1}$ and $\widetilde{P}_{2}$ are edge-disjoint. 


\section{B.1 Correctness}

The correctness of the main repeat-until loop in Algorithm 2ESCC-SPARSE apart from Procedure 2IsolatedSetLocal follows from the correctness of the basic algorithm for computing 2eSCCs. To show the correctness of Algorithm 2ESCC-SPARSE including Procedure 2IsolatedSetLocal, the following two parts are needed: (1) Every step in Procedure 2IsolatedSetLocal can be executed as described. (2) Whenever a non-empty set $S$ is identified by Procedure 2IsolatedSetLocal $(G, J)$, every 2eSCC of $G$ is completely contained in either $G[S]$ or $G[V \backslash S]$ and there are edges between $S$ and $V \backslash S$ in $G$. The latter property ensures that the algorithm terminates.

Let $\mathcal{G} \in\{G, \operatorname{Rev}(G)\}$. For Part (1) we need in particular that (1a) whenever we consider the flow graph $F_{\mathcal{G}, j}\left(r_{\mathcal{G}, j}\right)$, the set $B_{\mathcal{G}, j}$ contains at least one vertex; and that (1b) whenever there is an edge-dominator $e$ in $F_{\mathcal{G}, j}\left(r_{\mathcal{G}, j}\right)$, then there exists a tSCC without vertices of $B_{\mathcal{G}, j}$ in $\mathcal{G}_{j} \backslash\{e\}$. Lemma A.2 implies (1b). For (1a) note that if $B_{\mathcal{G}, j}=\emptyset$, we have that there are no edges from vertices of $V \backslash V_{\mathcal{G}, j}$ to vertices of $V_{\mathcal{G}, j}$ in $\mathcal{G}$. Thus in this case the subgraph $\mathcal{G}_{j}$ has to contain a tSCC.

Lemma B.2. If Procedure 2IsolatedSetLocal returns a non-empty set of vertices $S$, then (a) there exists an edge between vertices of $S$ and vertices of $V \backslash S$ in $G$ and (b) each $2 e S C C$ of $G$ is completely contained in either $G[S]$ or $G[V \backslash S]$.

Proof. Let $\mathcal{G} \in\{G, \operatorname{Rev}(G)\}$ be the graph in which the returned set is identified. Procedure 2IsolatedSetLocal returns a non-empty set for some $j \in J$ in three cases. It first searches for a set of vertices $T$ with $T \subseteq A_{\mathcal{G}, j}$ that induces a tSCC $\mathcal{G}[T]$ in $\mathcal{G}_{j}$. If the search is successful, it determines whether there exists an edge between vertices of $T$ and vertices of $V \backslash T$ in $G$. If this is satisfied, it returns the set $T$ (Case (1)). If this is not satisfied, i.e., $G[T]$ is a top and a bottom SCC in $G$, but $G[T]$ contains a bridge $e$, then the procedure returns a set of vertices $U$ that induces a top or bottom SCC $G[U]$ in $G[T] \backslash\{e\}$ (Case (2)). If $\mathcal{G}_{j}$ does not contain a tSCC without a vertex of $B_{\mathcal{G}, j}$, i.e., all vertices in $A_{\mathcal{G}, j}$ are reachable from some vertex of $B_{\mathcal{G}, j}$, then the procedure searches for an edge-dominator in $F_{\mathcal{G}, j}\left(r_{\mathcal{G}, j}\right)$; if an edge-dominator $e$ exists, the procedure returns a set of vertices $U$ with $U \subseteq A_{\mathcal{G}, j}$ that induces a tSCC $\mathcal{G}[U]$ in $\mathcal{G}_{j} \backslash\{e\}$ (Case (3)).

In Case (1) the existence of edges between vertices of $T$ and vertices of $V \backslash T$ is explicitly checked. By Lemma A.4 $\mathcal{G}[T]$ is a tSCC in $\mathcal{G}$. Since every $2 \mathrm{eSCC}$ is strongly connected, each 2eSCC of $G$ is completely contained in either $G[T]$ or $G[V \backslash T]$.

In Case $(2) \mathcal{G}[U]$ is an almost tSCC in $\mathcal{G}$ with respect to $e$ by the definition of a bridge. In Case (3) we have by Lemma A.3 and Corollary A.5 that $\mathcal{G}[U]$ is an almost tSCC in $\mathcal{G}$ with respect to $e$. Thus in both cases by Lemma A.1 no vertex of $V \backslash U$ has two edge-disjoint paths to any vertex of $U$ in $\mathcal{G}$. Hence each $2 \mathrm{eSCC}$ of $G$ has to be completely contained in either $G[U]$ or $G[V \backslash U]$. The identified edge $e$ has one endpoint in $V \backslash U$ and one in $U$, i.e., there exists at least one edge between vertices of $V \backslash U$ and vertices of $U$ in $G$.

Theorem B.3 (Correctness). Algorithm 2ESCC-SPARSE computes the 2eSCCs of the input graph.

Proof. Algorithm 2ESCC-SPARSE repeatedly removes edges from the input graph until the SCCs in the remaining graph correspond to the 2eSCCs of the input graph. We show the 
correctness of the algorithm by showing that (a) the removed edges cannot be in a 2eSCC, (b) when the algorithm terminates, each SCC is a 2eSCC, and (c) the algorithm terminates. By definition, 2eSCCs are strongly connected subgraphs that do not contain a bridge. Thus (a) clearly holds when edges between SCCs or bridges are removed in the repeat-until loop without the calls to 2IsolatedSetLocal. By Lemma B.2, (a) also holds for the edges removed after 2IsolatedSetLocal returns a non-empty set of vertices.

To show (b), first note that whenever 2IsolatedSetLocal is called, there will be another iteration of the repeat-until loop. This is because the algorithm terminates only in the case that $J$ is empty but 2IsolatedSetLocal is only called when there are vertices in $J$ and 2IsolatedSetLocal does not remove vertices from $J$. Consider the last iteration of the repeat-until loop. In this iteration no bridges were identified as otherwise $J$ cannot be empty. Thus no SCC in $G$ contains a bridge, i.e., each SCC in $G$ is a $2 \mathrm{eSCC}$.

For (c) we show that in each iteration of the inner and the outer-repeat until loop either edges are removed or the algorithm terminates. The inner repeat-until loop terminates when Procedure 2IsolatedSetLocal returns an empty set. Whenever Procedure 2IsolatedSetLocal returns a non-empty set $S$, by Lemma B.2 there exist edges between $S$ and $V \backslash S$ in $G$, which are then removed from $G$. For the outer repeat-until loop we have that the algorithm terminates if no bridges are identified in the for-loop; otherwise at least the bridges are removed from $G$.

\section{B.2 Runtime}

The next lemma applies the results of Appendix A.1 to the subgraphs used in Algorithm 2ESCC-SPARSE to show that we can indeed find all desired subgraphs with at most $d$ vertices in 2IsolatedSetLocal.

Lemma B.4. If Procedure 2IsolatedSetLocal $(G, J)$ returns an empty set, then

(a) each top or bottom SCC that is not disconnected from the remaining graph

(b) and each almost top or almost bottom SCC in $G$

that contains a vertex in $J$, has at least $d$ vertices.

Proof. We show the lemma by showing that if by contradiction an (almost) top or bottom SCC as described would exist, then Procedure 2IsolatedSetLocal $(G, J)$ would return a non-empty set.

Recall that $V_{\mathcal{G}, j}$ is the set of vertices that can reach a vertex $j$ in $\mathcal{G} \in\{G, \operatorname{Rev}(G)\}$ using a path containing at most $d$ edges and let $\mathcal{G}_{j}=\mathcal{G}\left[V_{\mathcal{G}, j}\right]$. Further recall that $B_{\mathcal{G}, j}$ is the set of vertices of $V_{\mathcal{G}, j}$ with incoming edges from vertices of $V \backslash V_{\mathcal{G}, j}$ and that $A_{\mathcal{G}, j}$ is equal to $V_{\mathcal{G}, j} \backslash B_{\mathcal{G}, j}$. The flow graph $F_{\mathcal{G}, j}\left(r_{\mathcal{G}, j}\right)$ is as in Definition A.7.

Assume there exists a set of vertices $S$ with $j \in S$ and $|S| \leq d$ that induces (a) a tSCC $\mathcal{G}[S]$ in $\mathcal{G}$ that has edges to vertices in $V \backslash S$ in $\mathcal{G}$ or (b) an almost tSCC $\mathcal{G}[S]$ in $\mathcal{G}$ with respect to some edge $e$. Since $|S| \leq d$ and $j \in S$, any simple path in $\mathcal{G}[S]$ from a vertex of $S \backslash\{j\}$ to $j$ can contain at most $d-1$ edges. Thus all vertices with incoming edges to vertices of $S$ in $\mathcal{G}$ can reach $j$ using a path with at most $d$ edges in $\mathcal{G}$. Hence $S$ is a subset of $V_{\mathcal{G}, j}$ and all incoming edges of $S$ are contained in $\mathcal{G}_{j}$, i.e., $S \subseteq A_{\mathcal{G}, j}$.

In Case (a) $\mathcal{G}[S]$ is a tSCC in $\mathcal{G}_{j}$ by Lemma A.4 and the procedure returns a non-empty set in Line 10. 
For Case (b) assume that no set is returned in Line 10, i.e., there does not exist a tSCC induced by a set of vertices $T$ in $A_{\mathcal{G}, j}$ in $\mathcal{G}_{j}$ such that there are edges from $T$ to $V \backslash T$ in $\mathcal{G}$. First consider the case that there exists a tSCC induced by a set of vertices $T$ in $A_{\mathcal{G}, j}$ in $\mathcal{G}_{j}$ such that there are no edges from $T$ to $V \backslash T$ in $\mathcal{G}$. In this case $T$ is a top and a bottom SCC in $G$ by Lemma A.4. Since all vertices in $V_{\mathcal{G}, j}$ are reachable from $j$, we have $T=V_{\mathcal{G}, j}$ and $B_{\mathcal{G}, j}=\emptyset$. Thus in this case the set $S$ is a proper subset of $T$ and the edge $e$ is a bridge in $\mathcal{G}[T]$. Hence Procedure 2IsolatedSetLocal $(G, J)$ returns a non-empty set in Line 14 .

Now consider the last case, namely assume that there does not exist a tSCC induced by a set of vertices $T$ in $A_{\mathcal{G}, j}$ in $\mathcal{G}_{j}$. Then all vertices in $A_{\mathcal{G}, j}$ are reachable from vertices of $B_{\mathcal{G}, j}$. Thus the edge $e$ is an edge-dominator in $F_{\mathcal{G}, j}\left(r_{\mathcal{G}, j}\right)$ by Corollary A.11 and the procedure returns a non-empty set in Line 19 .

In the runtime analysis we need that the algorithm indeed starts local searches in each top or bottom SCC or almost top or bottom SCC in the current graph $G$ that was not identified before. For this we use the following observation.

Observation B.5. Let $G$ be a directed graph. Let $X$ be a set of edges in $G$ and let $J$ be the set of vertices adjacent to an edge in $X$ in $G$. Let $H$ be a subgraph of $G$. Every $t S C C$ in $H \backslash X$ that has incoming edges in $H$ contains a vertex of $J$.

Proof. Let $S$ be a set of vertices that induces a tSCC in $H \backslash X$ that has incoming edges in $H$. Then the set of edges $X$ has to contain an incoming edge $(u, v)$ for some vertex $v \in S$. We have that $v$ is in $J$.

Theorem B.6 (Runtime). Algorithm 2ESCC-SPARSE can be implemented in time $O\left(m^{2} / \log n\right)$.

Proof. Let the input graph have $n$ vertices and $m$ edges. Recall that the input graph is converted to a constant degree graph with $n^{\prime}$ vertices and $m^{\prime}$ edges such that both $n^{\prime}$ and $m^{\prime}$ are of order $O(m)$. Let $G$ denote the (expanded) graph maintained by the algorithm.

Using the linear time algorithms to compute SCCs and find all bridges in a graph $G$, an iteration of the outer repeat-until loop without the calls to 2IsolatedSetLocal takes time $O\left(m^{\prime}\right)$. We will show that there can be only $O\left(\mathrm{~m}^{\prime} / q+n^{\prime} / d\right)$ iterations of the outer repeat-until loop, where $O\left(\mathrm{~m}^{\prime} / q+n^{\prime} / d\right)$ is $O\left(\mathrm{~m}^{\prime} / \log n^{\prime}\right)$. We will bound the time spent in the inner repeat-until loop separately.

A new iteration of the outer repeat-until loop is started in two cases.

Case 1: $|J| \geq q$. A vertex is in $J$ only when one of its adjacent edges was deleted from $G$ since the last time $J$ was initialized with the empty set. Thus Case 1 can happen at most $2 m^{\prime} / q$ times.

Case 2: 2IsolatedSetLocal returned an empty set. Let $G$ and $J$ be as maintained by the algorithm at the beginning of the subsequent iteration of the outer repeat-until loop. We distinguish two subcases. Let a subgraph $G[W]$ induced by some set of vertices $W$ be connected if for every partition of $W$ into two subsets there are edges between the subsets.

Case (2a): There exists a subgraph in $G$ that is connected but not strongly connected. Let $W$ be a set of vertices that induces a maximal connected but not strongly connected subgraph of $G$. Since $G[W]$ is connected, the vertices in $W$ were strongly connected when the edges between SCCs were removed at the beginning of the previous iteration of the outer repeat-until loop. As $G[W]$ is not strongly connected, it contains a top and a bottom SCC that are disjoint. By the maximality of $G[W]$, this top and this bottom SCC in $G[W]$ are 
also a top and a bottom SCC in $G$, respectively. By Observation B.5 each of them contains a vertex of $J$. Further, by Lemma B.4 each of them has more than $d$ vertices as otherwise at least one of them would have been identified by Procedure 2IsolatedSetLocal and thus they would not be connected in $G$. Both of them are identified when the SCCs of $G$ are determined and at least the outgoing edges of the tSCC and the incoming edges of the bSCC are removed from $G$. Thus in this case two vertex sets that each contain more than $d$ vertices are separated from each other by deleting all edges between them. This can happen at most $n^{\prime} / d$ times.

Case (2b): Every connected subgraph of $G$ is also strongly connected. After determining the SCCs of $G$, the algorithm searches for bridges in every SCC of $G$. If no bridge is found, the algorithm terminates. Assume that a bridge $e$ was found in an SCC $G[W]$ induced by some set of vertices $W$. The edge $e$ cannot have been a bridge when bridges were identified in the previous iteration of the outer repeat-until loop, as otherwise it would not be in $G$. Since $e$ is a bridge, there exist an almost top and an almost bottom SCC with respect to $e$ in $G[W]$. As every connected subgraph of $G$ is also strongly connected, there are no edges between $W$ and $V \backslash W$ in $G$. Thus the almost top and the almost bottom SCC in $G[W]$ are also an almost top and an almost bottom SCC in G. By Observation B.5 each of them contains a vertex of $J$. By Lemma B.4 each of them has more than $d$ vertices as otherwise the edge $e$ would have been identified by Procedure 2IsolatedSetLocal and removed from the graph. Thus in Case (2b) either the algorithm terminates or two vertex sets that each contain more than $d$ vertices and were strongly connected to each other are separated from each other by deleting the edge $e$ (such that they are no longer strongly connected but they might still be connected). This can happen at most $n^{\prime} / d$ times.

It remains to bound the time spent in 2IsolatedSetLocal. To this end note that each time before 2IsolatedSetLocal is called either (a) 2IsolatedSetLocal was called and a set of vertices $S$ that induces a top or bottom SCC $G[S]$ or an almost top or bottom SCC $G[S]$ in $G$ with respect to some edge $e$ was identified and separated from the remaining graph by deleting the edges between $S$ and $V \backslash S$ or (b) Bridges identified a bridge $e$ and increased the number of SCCs in $G$ by removing $e$. Both (a) and (b) can happen at most $n^{\prime}$ times.

We now consider the time for one call to 2IsolatedSetLocal. For each $j \in J$ this procedure runs a breadth-first search of depth $d$ on each of $\mathcal{G} \in\{G, \operatorname{Rev}(G)\}$ to identify the subgraphs $\mathcal{G}_{j}$. Considering $G$ and $\operatorname{Rev}(G)$ only increases the running time by a factor of two. The number of edges explored by a breadth-first-search of depth $d$ on a graph with out-degree at most three is $O\left(3^{d}\right)$. Thus with $d=\left\lceil\varepsilon \log n^{\prime}\right\rceil$ for some $0<\varepsilon<1$ we have that the number of edges in $\mathcal{G}_{j}$ is $O\left(\left(n^{\prime}\right)^{\varepsilon}\right)$. 2IsolatedSetLocal computes SCCs and bridges or edge-dominators in $\mathcal{G}_{j}$. This can be done in time linear in the number of edges in $G_{j}$, i.e., in time $O\left(\left(n^{\prime}\right)^{\varepsilon}\right)$. Thus with $|J|<q$ we obtain a time bound of $O\left(q \cdot\left(n^{\prime}\right)^{\varepsilon}\right)$ for one call to 2IsolatedSetLocal. Hence the total time spent in 2IsolatedSetLocal can be bounded with $O\left(q \cdot\left(n^{\prime}\right)^{\varepsilon} \cdot n^{\prime}\right)=O\left(\left(n^{\prime}\right)^{1+\varepsilon} \log n^{\prime}\right)$. We have that $O\left(\left(n^{\prime}\right)^{1+\varepsilon} \log n^{\prime}\right)$ is $O\left(m^{2} / \log n\right)$ for any $\epsilon \in(0,1)$. 


\section{Remark on relation to 2-edge strongly connected blocks}

The following construction shows that, in general, 2-edge strongly connected blocks do not provide any information about the 2-edge strongly connected components of a graph. Let $G=(V, E)$ be an arbitrary directed graph. We construct a graph $G^{\prime}$ by adding $O(|V|)$ edges and a constant number of vertices to $G$ such that all vertices in $V$ are in the same 2-edge strongly connected block in $G^{\prime}$, while the 2-edge strongly connected components in $G^{\prime}$ of the vertices in $V$ remain the same as in $G$. To construct $G^{\prime}$, we add to $G$ : the four vertices $s_{1}$, $t_{1}, s_{2}$, and $t_{2}$, the two edges $\left(s_{1}, t_{1}\right)$ and $\left(s_{2}, t_{2}\right)$, and for each vertex $v \in V$ the edges $\left(v, s_{1}\right)$, $\left(v, s_{2}\right),\left(t_{1}, v\right),\left(t_{2}, v\right)$. In $G^{\prime}$ each vertex $u \in V$ has two edge-disjoint paths to each vertex $v \in V$, namely the paths $\left(u, s_{1}, t_{1}, v\right)$ and $\left(u, s_{2}, t_{2}, v\right)$. Thus all vertices of $V$ are in the same 2-edge strongly connected block in $G^{\prime}$. However, clearly the edges $\left(s_{1}, t_{1}\right)$ and $\left(s_{2}, t_{2}\right)$ are bridges in $G^{\prime}$. Thus the 2 -edges strongly connected components in $G^{\prime}$ are the same as in $G$ (plus the trivial subgraphs induced by each of the newly added vertices).

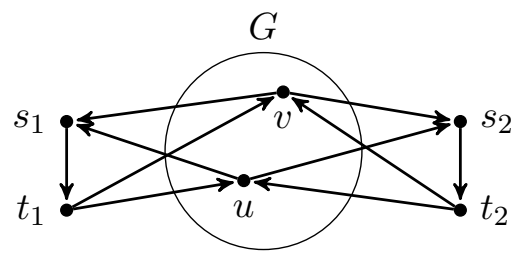

